\title{
Transactivation of RAGE mediates angiotensin-induced inflammation and atherogenesis
}

\author{
Raelene J. Pickering, ${ }^{1,2}$ Christos Tikellis, ${ }^{1,2}$ Carlos J. Rosado, ${ }^{1,2}$ Despina Tsorotes, ${ }^{2}$ Alexandra Dimitropoulos, ${ }^{1}$ Monique Smith, ${ }^{1}$ \\ Olivier Huet, ${ }^{2,3}$ Ruth M. Seeber, ${ }^{4}$ Rekhati Abhayawardana,, ${ }^{4}$ Elizabeth K.M. Johnstone, ${ }^{4}$ Jonathan Golledge, ${ }^{5}$ Yutang Wang, ${ }^{5}$ \\ Karin A. Jandeleit-Dahm, ${ }^{1,2}$ Mark E. Cooper, ${ }^{1,2}$ Kevin D.G. Pfleger, ${ }^{4,6}$ and Merlin C. Thomas ${ }^{1,2}$ \\ 'Department of Diabetes, Central Clinical School, Monash University, Melbourne, Australia. ${ }^{2}$ Baker IDI Heart and Diabetes Institute, Melbourne, Australia. ${ }^{3}$ Department of Anaesthesia and Intensive Care, \\ Centre Hospitalier Régional Universitaire (CHRU) La Cavale Blanche, Universite de Bretagne Ouest, Brest, France. ${ }^{4}$ Molecular Endocrinology and Pharmacology, Harry Perkins Institute of Medical Research \\ and Centre for Medical Research, The University of Western Australia, Nedlands, Australia. ${ }^{5}$ The Vascular Biology Unit, Queensland Research Centre for Peripheral Vascular Disease, James Cook University, \\ Townsville, Australia. ${ }^{6}$ Dimerix Limited, Nedlands, Western Australia, Australia.
}

\begin{abstract}
Activation of the type 1 angiotensin II receptor $\left(\mathrm{AT}_{1}\right)$ triggers proinflammatory signaling through pathways independent of classical Gq signaling that regulate vascular homeostasis. Here, we report that the $\mathrm{AT}_{1}$ receptor preformed a heteromeric complex with the receptor for advanced glycation endproducts (RAGE). Activation of the AT, receptor by angiotensin II (Ang II) triggered transactivation of the cytosolic tail of RAGE and NF-KB-driven proinflammatory gene expression independently of the liberation of RAGE ligands or the ligand-binding ectodomain of RAGE. The importance of this transactivation pathway was demonstrated by our finding that adverse proinflammatory signaling events induced by AT $_{1}$ receptor activation were attenuated when RAGE was deleted or transactivation of its cytosolic tail was inhibited. At the same time, classical homeostatic Gq signaling pathways were unaffected by RAGE deletion or inhibition. These data position RACE transactivation by the AT, receptor as a target for vasculoprotective interventions. As proof of concept, we showed that treatment with the mutant RAGE peptide S391A-RAGE ${ }_{362-404}$ was able to inhibit transactivation of RAGE and attenuate Ang II-dependent inflammation and atherogenesis. Furthermore, treatment with WT RACE ${ }_{362-404}$ restored Ang II-dependent atherogenesis in Ager/Apoe-KO mice, without restoring ligand-mediated signaling via RAGE, suggesting that the major effector of RAGE activation was its transactivation.
\end{abstract}

\section{Introduction}

Blockade of the renin-angiotensin aldosterone system (RAAS) is one of the most prescribed treatments in all of medicine. The pleiotropic benefits of RAAS blockade on inflammation (1-3), atherogenesis $(4,5)$, and even longevity (6) are additional to and independent of blood pressure lowering (7). Activation of the type 1 angiotensin II receptor ( $\mathrm{AT}_{1}$ ) receptor by angiotensin II (Ang II) triggers pathogenic pathways that are distinct from classical $\mathrm{Gq}$ protein signaling that induces vasoconstriction and sodium retention $(2,3,8,9)$. However, a complete understanding of this fundamental division and, more important, of any means to specifically block the proinflammatory effects of Ang II remains elusive.

The receptor for advanced glycation endproducts (RAGE) is a type I transmembrane glycoprotein. RAGE is basally expressed at high levels in the vascular endothelium and leukocytes, although

\section{Authorship note: RJP, CT, and CJR are co-first authors.}

Conflict of interest: KDCP is chief scientific adviser of Dimerix Limited, a spin-off company of The University of Western Australia that has been assigned the rights to Receptor-HIT. KDGP is an inventor on patents covering the technology (World Intellectual Property [WIPO] patent no. WO/2008/055313, held by Dimerix Limited) and is a shareholder of Dimerix Limited.

License: Copyright 2019, American Society for Clinical Investigation.

Submitted: January 23, 2018; Accepted: October 30, 2018.

Reference information: J Clin Invest. 2019;129(1):406-421.

https://doi.org/10.1172/JCI99987.
RAGE expression may be induced in most cell types following injury, inflammation, or hypoxia (10). RAGE is implicated in a range of diseases, including Alzheimer's disease (11), malignancy (12), diabetic complications (13), and atherosclerosis (14-17). In each case, dysfunction has been ascribed to activation of the RAGE ectodomain by ligands including S100 proteins (also known as calgranulins) and advanced glycation endproducts (AGEs) that trigger $\mathrm{NF}-\kappa \mathrm{B}$-driven proinflammatory gene expression (18). However, we provide evidence for a cognate ligand-independent mechanism for RAGE transactivation that occurs following activation of the $\mathrm{AT}_{1}$ receptor. Using a variety of mouse models, assay systems, and different RAGE domains, we show that the receptors form a plasma membrane-localized heteromeric complex, which, upon activation, induces $\mathrm{NF}-\kappa \mathrm{B}$ expression and the subsequent canonical and noncanonical signaling that largely determines the proinflammatory effects of RAAS activation.

\section{Results}

Atherogenesis following an infusion of Ang II is reduced in Ager/ Apoe-double-KO mice. To demonstrate the role of RAGE in Ang II-dependent atherogenesis, we infused atherosclerosis-susceptible Apoe-KO mice with Ang II $(1 \mu \mathrm{g} / \mathrm{kg} / \mathrm{min})$ for 4 weeks. This resulted in increased plaque accumulation across the aortic arch in Apoe-KO but not Ager/Apoe-double-KO (Ager/Apoe-DKO) mice (Figure 1A). Exposure to Ang II also increased aortic expression 

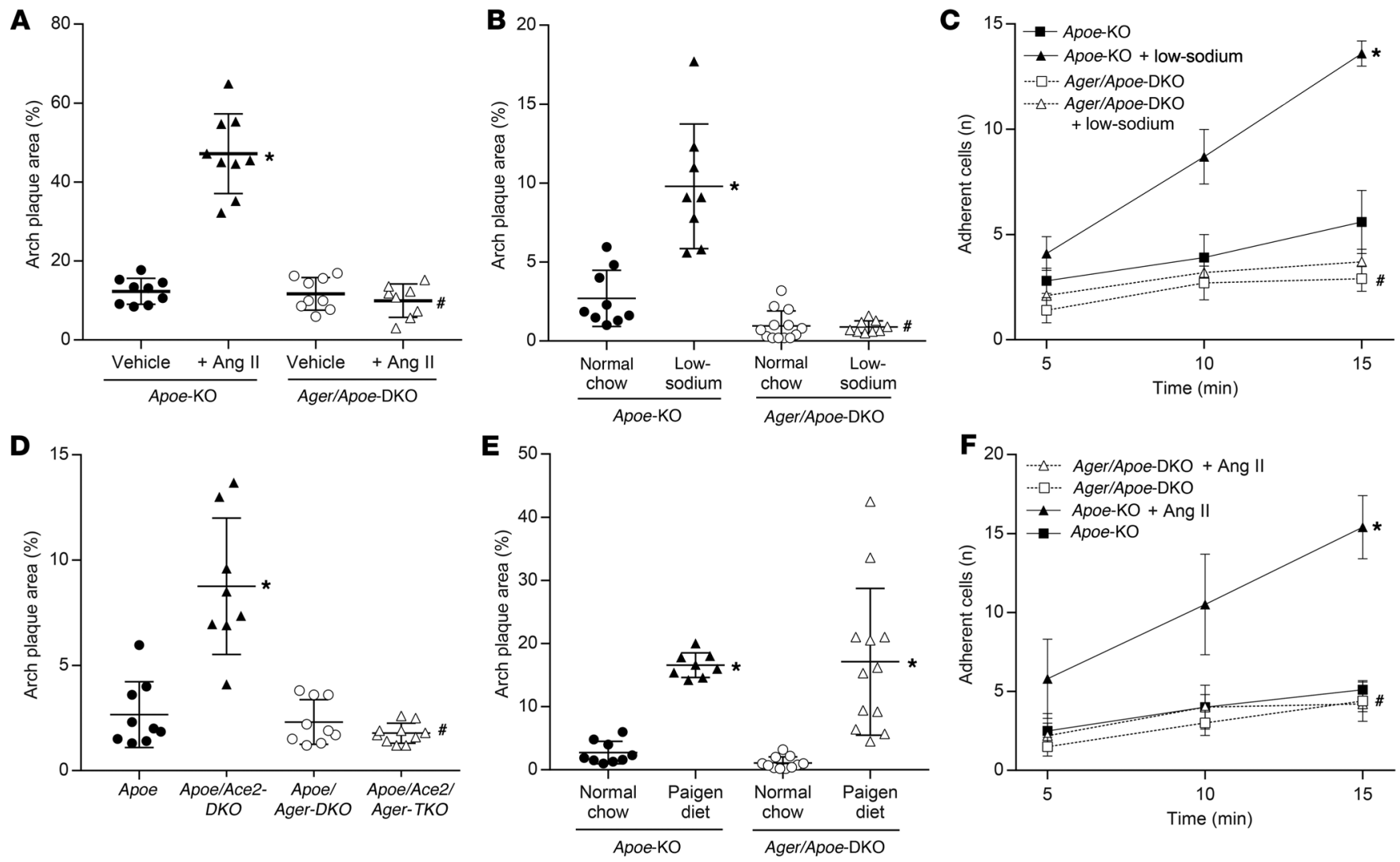

Figure 1. Activation of the RAAS is associated with increased atherogenesis and endothelial activation in Apoe-KO but not Ager/Apoe-DKO mice. (A) Increased plaque area following a 4-week infusion of Ang II (1 $\mu \mathrm{g} / \mathrm{kg} / \mathrm{min})$. (B) Increased plaque area following 6 weeks of a $0.05 \%$ low-sodium diet. (C) Increased number of labeled leukocytes adhering to the aortic surface as a marker of endothelial activation after 1 week of exposure to a low-sodium diet. (D) Increased plaque area in the presence of genetic Ace2 deficiency. (E) Equivalent increase in plaque area following exposure to a Paigen proatherogenic diet. (F) Increased number of labeled leukocytes adhering to the aortic surface following ex vivo exposure to Ang II (1 $\mu \mathrm{M}$ for $4 \mathrm{~h})$. Data are presented as the mean \pm SD. $n=8-10$ mice per group. ${ }^{*} P<0.05$ versus Apoe-KO mice; ${ }^{\#} P<0.05$ versus Apoe-KO mice plus RAAS activation (Ang II infusion, low-sodium diet, Ace2/Apoe-DKO). $P$ values were determined by 2-way ANOVA.

of proatherogenic mediators (Supplemental Figure 1A; supplemental material available online with this article; https:// doi.org/10.1172/JCI99987DS1) in Apoe-KO mice but not Ager/ Apoe-DKO mice. However, we found that systolic blood pressure increased to a similar extent in response to Ang II in both the presence and absence of RAGE (Supplemental Table 1), and neither Ang II nor RAGE deficiency modified total cholesterol levels (no significant difference, $P>0.1$ ).

Atherogenesis following a low-sodium diet is reduced in Ager/ApoeDKO mice. In a second model of Ang II-dependent atherogenesis, 6 weeks of exposure to a $0.05 \%$ (low) sodium diet also resulted in RAAS-dependent plaque accumulation in Apoe-KO mice (Figure 1B) and increased aortic expression of proatherogenic mediators (Supplemental Figure 1B). More labeled leukocytes were adherent to the endothelium of aortae taken from Apoe-KO mice after 1 week of exposure to a low-sodium diet when compared with aortae from Apoe-KO mice fed a normal chow diet (Figure 1C). In contrast, we observed that the same low-sodium diet fed to Ager/Apoe-DKO mice did not modify plaque accumulation (Figure 1B) or markers of vascular inflammation (Supplemental Figure 1B), despite comparable activation of the systemic RAAS and sodium retention (Supplemental Figure 1, E-G). Additionally, we found that Ager/Apoe-DKO mice fed a low-sodium diet did not have increased endothelial adhesion of labeled leukocytes (Figure 1C). Blood pressure levels were not reduced by a low-sodium diet in either Apoe-KO or Ager/Apoe-DKO mice (Supplemental Table 1), and a low-sodium diet had no effect on total cholesterol levels (no significant difference, $P>0.1$ ).

Atherogenesis in Ace2/Apoe-DKO mice is reduced in Ager/Ace2/ Apoe-triple-KO mice. Atherosclerosis was also explored in a third model of Ang II-dependent atherogenesis, this time using Ace2/ Apoe-DKO mice. We found that genetic deficiency of Ace 2 in Apoe-KO mice was associated with increased circulating levels of Ang II (Supplemental Figure 1H), elevated systolic blood pressure (Supplemental Table 1), and augmented plaque accumulation (Figure 1D), as previously described (19). The aortae of Ace2/ Apoe-DKO mice also had increased expression of proatherogenic mediators (Supplemental Figure 1C) when compared with aortae of Apoe-KO mice. In contrast, the induction of proinflammatory markers (Supplemental Figure 1C) and plaque accumulation (Figure 1D) were attenuated in Ager/Ace2/Apoe-triple-KO (Ager/ Ace2/Apoe-TKO) mice, despite a comparable elevation in circulating Ang II levels (Supplemental Figure 1H) and blood pressure (Supplemental Table 1), and Ace2 deficiency had no effect on total cholesterol (no significant difference, $P>0.1$ ). 
While RAGE deletion attenuated atherogenesis in RAASdependent models, it could be argued that this was due to nonspecific antiatherogenic actions of RAGE deletion. However, after 6 weeks of a cholate-containing high-fat (Paigen) diet, a lipiddependent model of atherosclerosis (20), we found that aortic plaque accumulation was increased in both Apoe-KO and Ager/ApoeDKO mice, with no significant difference between them (Figure 1E).

The induction of proinflammatory mediators by Ang II is dependent on RAGE expression. In isolated aortae from Apoe-KO mice, ex vivo exposure to Ang II $(1 \mu \mathrm{M})$ also increased the expression of proatherogenic mediators (Supplemental Figure 1D) and increased the number of adherent leukocytes when compared with untreated aortae (Figure $1 \mathrm{~F}$ ). In contrast, ex vivo treatment of aortae taken from Ager/Apoe-DKO mice with Ang II did not induce these atherogenic mediators (Supplemental Figure 1D) or increase leukocyte adhesion (Figure $1 \mathrm{~F}$ ).

In primary murine aortic endothelial cells (PMAECs) isolated from the aortae of C57BL/6J mice, exposure to Ang II resulted in a rapid increase in hydrogen peroxide (Figure 2A). This was associated with the induction of canonical and noncanonical signaling via NF- $\kappa \mathrm{B}$-dependent pathways (Figure 2B), leading to increased expression of key proinflammatory genes (Figure $2 \mathrm{C}$ ) and, functionally, the induction of a proatherogenic phenotype associated with increased adhesion of labeled THP-1 cells to a monolayer of Ang II-treated PMAECs (Figure 2D). We did not observe these Ang II-induced changes in PMAECs isolated from Ager-KO mice. Likewise, silencing of Ager or NF-kB-p65 expression in PMAECs using siRNA (Supplemental Figure 2, A-C) also prevented the $\mathrm{NF}-\mathrm{\kappa B}$-dependent induction of ICAM-1 by Ang II or the RAGE ligand S100A8/A9 (Figure 2E). The observed attenuation of Ang II effects in PMAECs from Ager-KO mice was not due to a loss of RAAS-signaling capacity in the absence of RAGE, as exposure of RAGE-deficient PMAECs to Ang II led to a similar increase in inositol monophosphate (IP $)$ (Figure $2 \mathrm{~F}$ ) and downstream induction of the early growth response gene Egr1 (Supplemental Figure $3 \mathrm{~A})$. In addition, we found that the classical induction of canonical $\mathrm{NF}-\kappa \mathrm{B}$ signaling by TNF- $\alpha$ was not attenuated in PMAECs from Ager-KO mice (Figure 2B). The role of RAGE in Ang II-induced proinflammatory signaling was not specific to endothelial cells and was also observed in Ang II-induced activation of macrophages or splenocytes (Supplemental Figure 4, A-C).

To specifically explore the signaling interaction between RAGE and the $\mathrm{AT}_{1}$ receptor, we transfected $\mathrm{CHO}$ cells with AGTR1 and $A G E R$, alone or in combination and observed that expression of the $\mathrm{AT}_{1}$ receptor in $\mathrm{CHO}$ cells conferred responsiveness to exogenous Ang II, as demonstrated by the induction of inositol phosphate synthesis (Figure 3A). This was not modified by the coexpression of full-length RAGE. In contrast, NF- $\kappa \mathrm{B}$ activation and induction of $p 65$ gene expression following exposure to Ang II in $\mathrm{AT}_{1}$ - $\mathrm{CHO}$ cells was dependent on RAGE coexpression (Figure $3 \mathrm{~B})$. Similar to this, RAGE expression was also required for the proinflammatory response to the RAGE ligand S100A8/A9 in CHO cells (Figure 3C) and PMAECs (Supplemental Figure 5A).

Ligand-independent transactivation of RAGE following activation of the $A T_{1}$ receptor by Ang II. Activation of the $\mathrm{AT}_{1}$ receptor is known to trigger transactivation of tyrosine kinase receptors, with one proposed mechanism being protease-mediated extracellular "shedding" of membrane-tethered ligands (21). Activation of the RAAS increased circulating concentrations of RAGE ligands in mice, including S100A8/A9 and AGEs and their reactive dicarbonyl precursors (Supplemental Figure 4, D-F), as well as increased expression of $\mathrm{Mac1}$ (also known as CD11b) in the aorta (Supplemental Figure 1, A-C). However, Ang II-induced proinflammatory signaling was not inhibited by pretreatment with a neutralizing antibody targeting the extracellular ligand-binding domain of RAGE (RAGEab), although RAGE ligand-dependent signaling was fully blocked by this strategy (Figure 3D and Supplemental Figure 5B). Likewise, deletion of the ligand-binding extracellular domain of RAGE did not modify the ability of N-truncated RAGE constructs to mediate Ang II-dependent induction of p65 expression or $\mathrm{NF}-\kappa \mathrm{B}$ activity in $\mathrm{AT}_{1}-\mathrm{CHO}$ cells, such that full-length RAGE activity in this assay was retained even by RAGE $_{370-404}$ (Figure $3 \mathrm{E}$ ). In contrast, the induction of $p 65$ expression in $\mathrm{AT}_{1}-$ $\mathrm{CHO}$ cells by $\mathrm{S100A8/A9}$ was lost in cells expressing N-truncated RAGE constructs in which the ligand-binding ectodomain was also deleted (Supplemental Figure 3B).

No role for the phosphorylation of RAGE following activation of the $A T_{1}$ receptor. The human RAGE cytosolic tail contains 4 potential phosphorylation sites, only 1 of which (S391) is partially conserved across other mammals (Supplemental Table 2). We observed that mutation of S399, S400, or T401 to alanine had no effect on signaling (Supplemental Figure 3C) and that signaling was also retained in E392X-RAGE (Figure 3F), despite its lack of 13 C-terminal residues (E392-P404). In contrast, full-length or C-truncated RAGE constructs in which S391 was deleted (S391XRAGE) (Figure 3F) or changed to alanine (S391A-RAGE) (Figure $4 \mathrm{~A}$ ) had no ability to mediate proinflammatory signaling in response to Ang II in $\mathrm{AT}_{1}$ - $\mathrm{CHO}$ cells. However, despite lacking a serine at 391, full-length S391Q-RAGE and S391P-RAGE mutants retained the ability to signal in response to Ang II and S100A8/A9 in $\mathrm{AT}_{1}$-CHO cells (Figure $4 \mathrm{~B}$ ). This was not through redundancy offered by other potential phosphorylation sites, as replacement of the 14 C-terminal amino acids in human RAGE (SEEPEAGESSTGGP) with those found in murine RAGE (SEEAEMPENGAGGP) to create a chimeric RAGE (cRAGE), with or without additional S391Q mutation (i.e., QEEAEMPENGAGGP), did not prevent signaling in response to Ang II or S100A8/A9 (Figure 4C). Moreover, mutation of the C-terminal to further remove potentially phosphomimetic residues (glutamate or aspartate) in addition to all serines and threonines (i.e., PKGPQAGQGGAGGP; no STED) also did not prevent Ang II-dependent signaling via RAGE in $\mathrm{AT}_{1}$ CHO cells (Supplemental Figure 3D).

Although phosphorylation of RAGE was not required for sig-

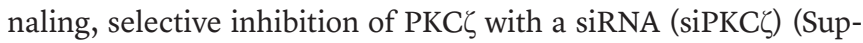
plemental Figure 2D) or a $\mathrm{PKC} \zeta$ pseudosubstrate (PKC $\zeta \mathrm{i})$ prevented the proinflammatory signaling induced by S100A8/A9 and Ang II in PMAECs (Figure 4D). We also observed the inhibitory effects of $\mathrm{PKC} \zeta \mathrm{i}$ in $\mathrm{AT}_{1}-\mathrm{CHO}$ cells expressing S391Q-cRAGE, despite the absence of serine/threonine targets for phosphorylation in this RAGE construct (Figure 4E). Silencing of PKC $\zeta$ expression (Supplemental Figure 2E) also inhibited signaling induced in human microvascular endothelial cells (HMEC1) (Figure 4F), which was rescued by overexpression of the purely cytosolic RAGE ${ }_{362-404}$ but not of the membrane-bound RAGE ${ }_{343-404}$ (Figure 4F). 

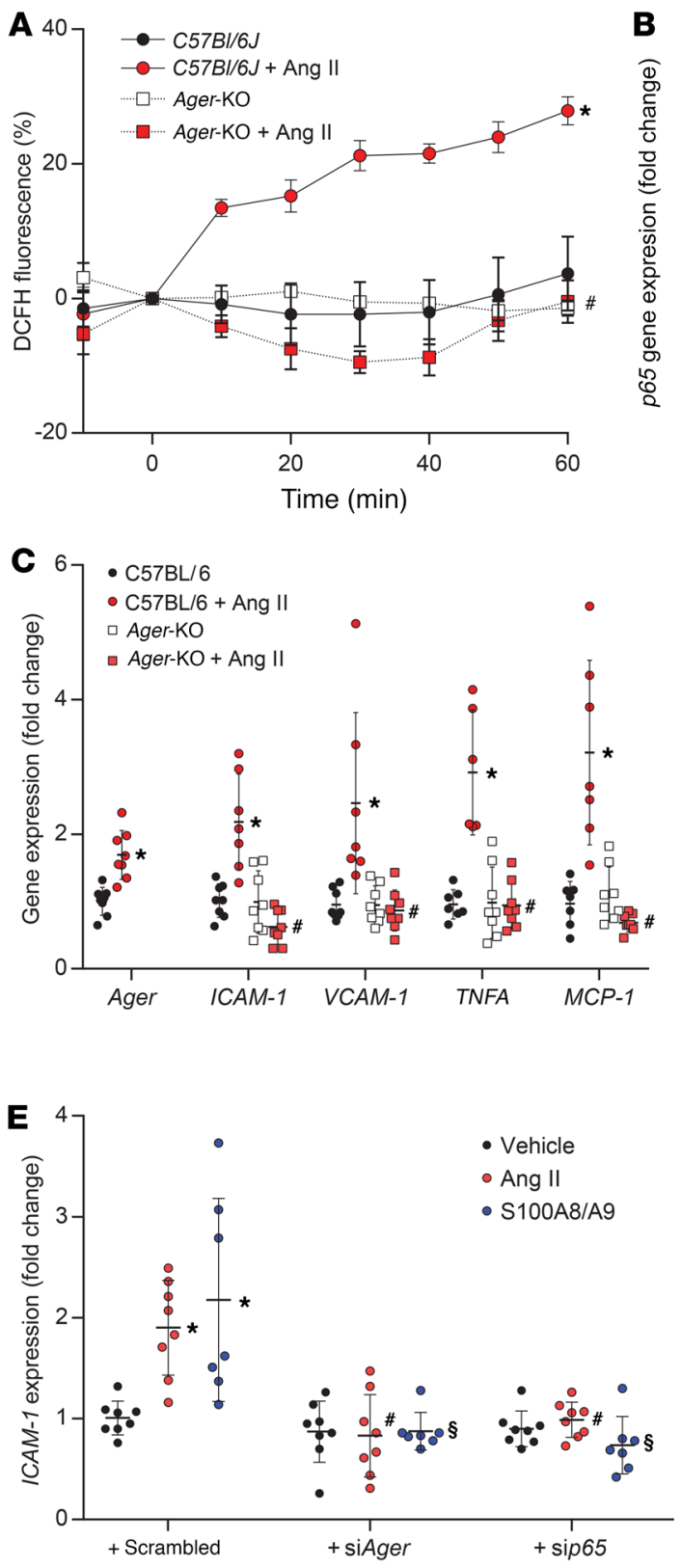
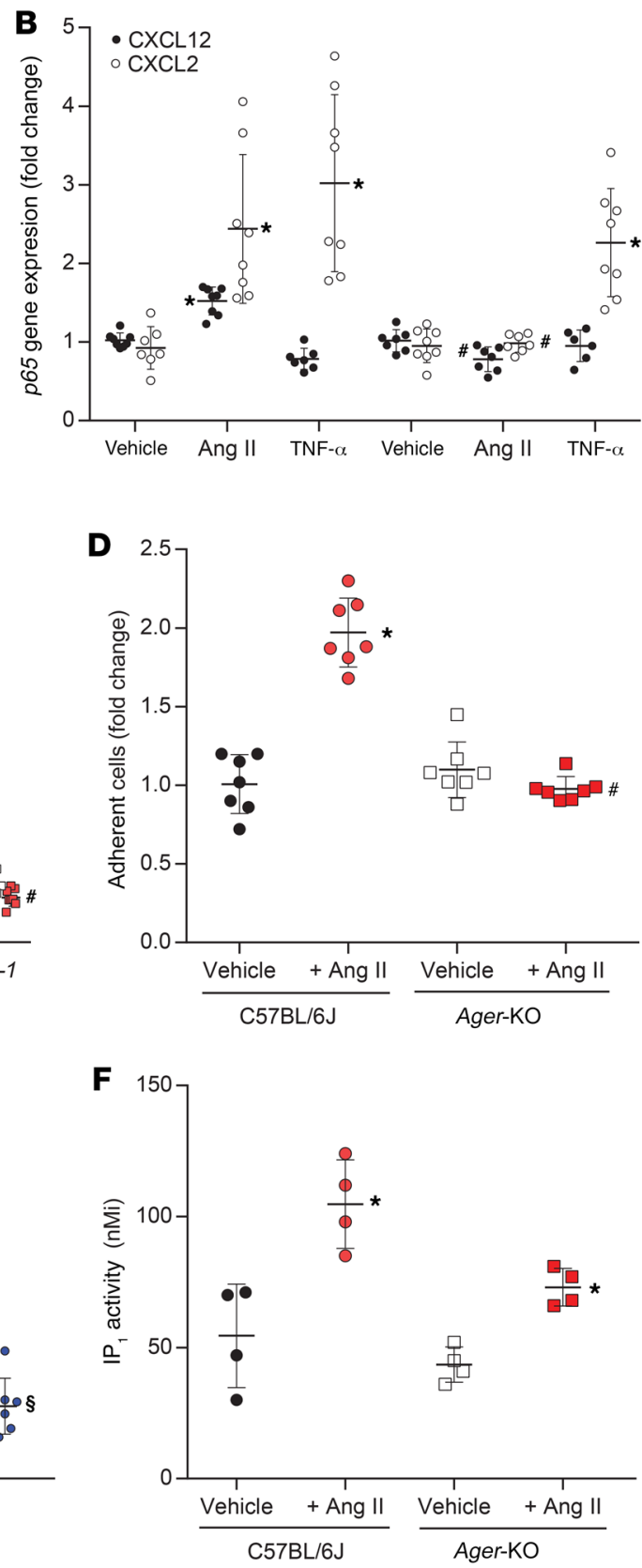

Figure 2. Differential effects of exposure to Ang II ( $1 \mu \mathrm{M})$ in PMAECs from C57BL/6] or Ager-KO mice. (A) Induction of hydrogen peroxide production in a flow chamber. (B) Induction of the canonical and noncanonical $\mathrm{NF}-\kappa \mathrm{B}$ signaling gene markers CXCL12 and CXCL2, respectively, following exposure to Ang II or the prototypical NF- $\kappa \mathrm{B}$-inducing cytokine TNF- $\alpha$ (1 ng/ml for $24 \mathrm{~h}$ ). (C) Induction of gene expression of proatherogenic mediators, including adhesion molecules, inflammatory chemokines, and cytokines, following exposure to Ang II (1 $\mu \mathrm{M}$ for $2 \mathrm{~h})$. (D) Quantitation of labeled THP-1 monocytes adherent to a monolayer of PMAECs from [57BL/6] and Ager-KO mice with or without Ang Il pretreatment ( $1 \mu \mathrm{M}$ for $2 \mathrm{~h})$. (E) Induction of ICAM-1 expression by Ang II ( $1 \mu \mathrm{M}$ for $2 \mathrm{~h})$ and the RAGE ligand S100A8/A9 $(2 \mu \mathrm{g} / \mathrm{ml}$ for 2 h) following silencing of RAGE or p65 expression by an siRNA (see Supplemental Figure 2, A-C, for details). (F) Induction of classical Gq inositol phosphate signaling, as estimated by the accumulation of $I P_{1}$ at 2 hours. Data are presented as the mean \pm SD. $n=6-8$ per group. ${ }^{*} P<0.05$ versus [57BL/6] ${ }^{\#} P<0.05$ versus $[57 \mathrm{BL} / 6$ ] plus Ang II; ${ }^{\S} P<0.05$ versus $[57 \mathrm{BL} / 6$ ] plus S100A8/A9. $P$ values were determined by 2-way ANOVA.
Role of diaphanous-1 in signaling via RAGE. The formin-type protein diaphanous-1 (Diaph1) is regarded as a key element for proinflammatory signaling induced by RAGE ligands (22). Consistent with these data, we observed that silencing of Diaph1 expression (Supplemental Figure 2F) prevented the induction of key proinflammatory genes in response to the RAGE ligand S100A8/9 in PMAECs as well as RAGE ligand-independent induction of ICAM-1 (Figure 4, F and G) and leukocyte adhesion to an endothelial monolayer (Supplemental Figure 2H) following exposure to Ang II. Diaph1 and RAGE are thought to interact specifically via a charged patch formed by R366 and Q367 (22), and we observed that R366A-Q367A-RAGE constructs were not able to mediate S100-induced signaling in $\mathrm{AT}_{1}-\mathrm{CHO}$ cells (Figure $4 \mathrm{H}$ ), as previously reported (22). In contrast, the R366A-Q367A-RAGE mutation did not alter the ability of full-length or N-truncated RAGE constructs to mediate the proinflammatory signaling induced by Ang II in $\mathrm{AT}_{1}-\mathrm{CHO}$ cells (Figure $4 \mathrm{H}$ ). Indeed, the transactivation of a construct lacking this domain $\left(\mathrm{RAGE}_{370-404}\right)$ following treatment with Ang II in $\mathrm{AT}_{1}-\mathrm{CHO}$ cells (Figure $3 \mathrm{E}$ ) suggests that, unlike RAGE ligand-dependent signaling, this domain is not essential for RAGE ligand-independent signaling. Nonetheless, the finding that silencing of Diaph1 expression in HMEC1 inhibited signaling by endogenous RAGE (Figure 4G) and membrane-bound RAGE $_{343-404}$ (Figure 4F) demonstrates that Diaph1 still plays a critical role. The finding that silencing of Diaph1 expression did not inhibit transactivation of the purely cytosolic construct RAGE ${ }_{362-404}$ (Figure 4F) suggests that, like PKC , a key target of Diaph1 may also be the single transmembrane domain of RAGE.

Bioluminescence resonance energy transfer indicates close proximity of the $A T_{1}$ receptor and RAGE when coexpressed in live cells. Con- 
A
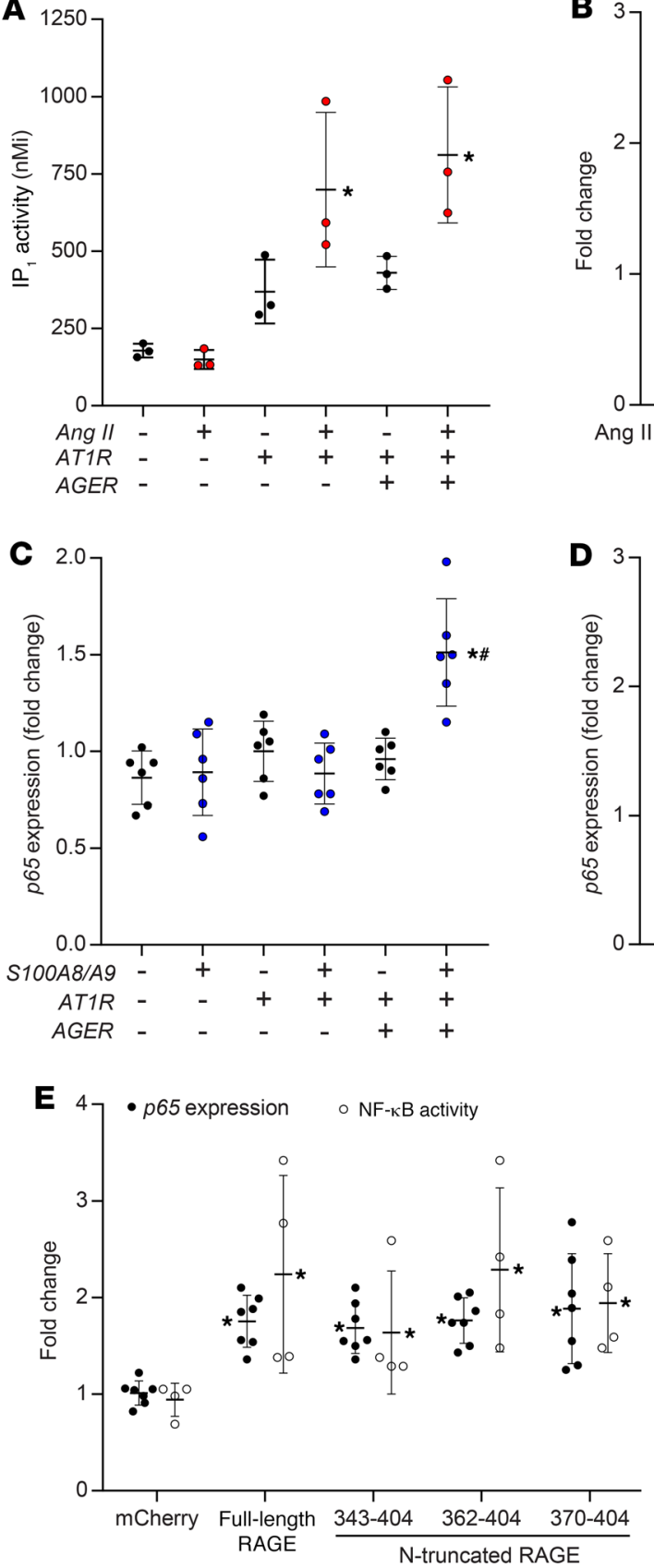
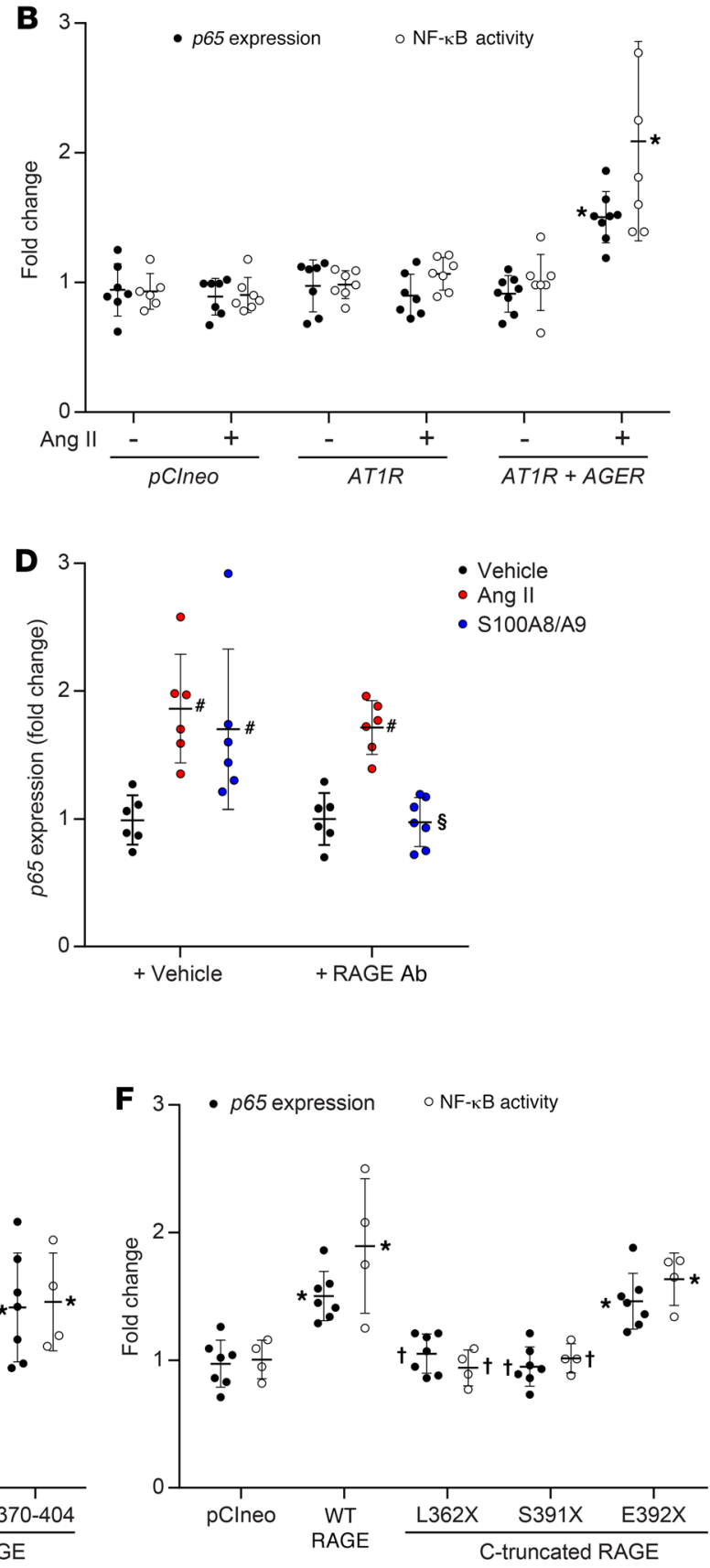

Figure 3. Functional response to Ang II or S100A8/A9 in the presence or absence of the human AT, receptor, RACE constructs, or both. Treatment with Ang II was at a dose of $1 \mu \mathrm{M}$ for 2 hours, and treatment with S100A8/A9 was at a dose of $2 \mu \mathrm{g} / \mathrm{ml}$ for 2 hours. (A) Induction of inositol phosphate synthesis. (B) NF- $\kappa B$ activation as measured by a chemiluminescent SEAP reporter gene assay, and induction of gene expression of the NF- $\kappa B$ subunit $p 65$ in the presence or absence of full-length human RAGE. (C) Induction of $p 65$ expression following exposure to the RAGE ligand, S100A8/A9 ( $2 \mu \mathrm{g} / \mathrm{ml}$ for $2 \mathrm{~h})$ in the presence or absence of full-length RAGE. (D) Induction of $p 65$ following exposure to S100A8/A9 or Ang II in PMAECs in the presence or absence of soluble RAGE or a neutralizing antibody targeting the extracellular domain of RAGE. (E) NF- $\kappa B$ activation and the induction of $p 65$ expression in the presence or absence of full-length or N-truncated RAGE constructs in AT - $\mathrm{CHO}$ cells. (F) NF- $\kappa B$ activation and the induction of $p 65$ expression by Ang II in the presence or absence of full-length or C-truncated RAGE constructs in AT - -CHO cells. Data are presented as the mean \pm SD. $n=6-8$ per group. ${ }^{*} P<0.05$ versus AT -

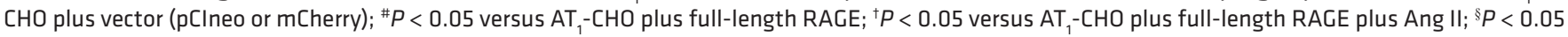
versus AT - -CHO plus RAGE plus S100A8/A9. $P$ values were determined by 2 -way ANOVA.

sistent with the functional interactions between RAGE and the $\mathrm{AT}_{1}$ receptor detailed above, coexpression of the Rluc8-labeled $\mathrm{AT}_{1}$ receptor ( $\mathrm{AT}_{1}$-Rluc8) and Venus-labeled RAGE (RAGE-Venus) in HEK293FT cells resulted in the generation of a strong and saturable bioluminescence resonance energy transfer (BRET) signal indicative of their close proximity, which was reduced upon treatment with Ang II (Figure 5A). Ang II treatment in cells coexpressing $\mathrm{AT}_{1}-\mathrm{Rluc}_{\mathrm{C}}$ and the signaling and regulatory protein $\beta$-arrestin2 tagged with Venus induced a robust and stable ligand-induced BRET signal consistent with recruitment of $\beta$-arrestin2 to the $\mathrm{AT}_{1}$ 

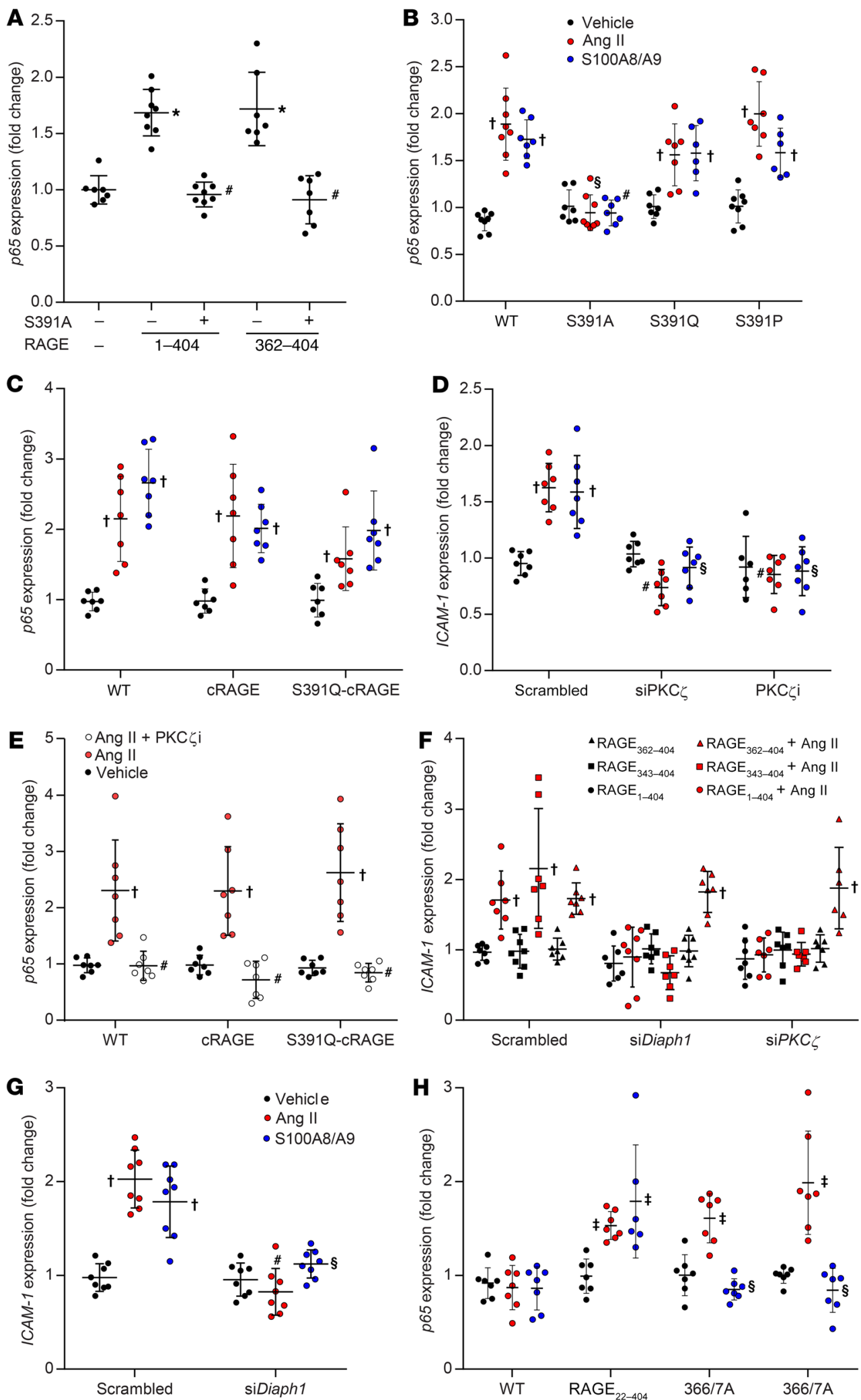

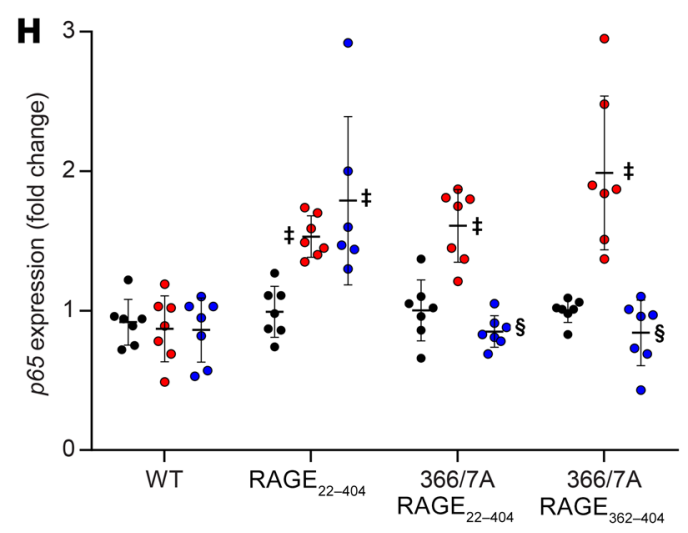

Figure 4. Modulation of proinflammatory signaling induced via RAGE activation by Ang II and/or S100A8/ A9. Treatment with Ang II was at a dose of $1 \mu \mathrm{M}$ for 2 hours, and treatment with S100A8/A9 was at a dose of $2 \mu \mathrm{g} / \mathrm{ml}$ for 2 hours. (A) Induction of $p 65$ expression in $\mathrm{AT}_{1}$ - $\mathrm{CHO}$ cells by Ang II in the presence or absence of full-length and N-truncated RACE constructs in which $\mathrm{S} 391$ was mutated to alanine (S391A). (B) Induction of $p 65$ expression in $\mathrm{AT}_{1}$ - $\mathrm{CHO}$ cells in the presence or absence of full-length RAGE constructs in which $\mathrm{S} 391$ was mutated. (C) Induction of $p 65$ expression in AT $\mathrm{CHO}$ cells in the presence or absence of cRAGE constructs. (D) Induction of ICAM-1 expression in PMAECs in the presence or absence of a siRNA against $\mathrm{PKC}$, a $\mathrm{PKC} \zeta$ pseudosubstrate

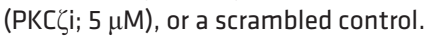
(E) Induction of $p 65$ expression in $\mathrm{AT}_{1}$ CHO cells also expressing WT or CRAGE constructs in the presence or absence of PKC $\zeta$ i. (F) Induction of ICAM-1 expression in HMEC1 in the presence or absence of a siRNA targeting $P K C \zeta$ or Diaph1 expression, and the rescue of inhibition by RAGE $\mathrm{B}_{362-404}$ but not RAGE $_{343-404^{\circ}}$ (C) Induction of ICAM-1 expression in the presence or absence of a siRNA targeting Diaph1 expression in HMEC1. (H) Induction of $p 65$ expression in AT - $\mathrm{CHO}$ cells in the presence or absence of full-length and N-truncated 366/367A-RAGE constructs. Data are presented as the mean \pm SD. $n=6-8$ group. ${ }^{*} P<0.05$ versus mCherry plus Ang II; ${ }^{P} P<0.05$ versus RAGE (transfected into AT1-CHO cells or endogenous in HMEC1) plus Ang II treatment; ${ }^{\dagger} P<0.05$ versus vehicle; $\$ P<0.05$ versus RAGE (transfected into AT1-CHO cells or endogenous in HMEC1) plus S100A8/A9; ${ }^{\ddagger} P<0.05$ versus $\mathrm{pCINeo}$ plus vehicle. $P$ values were determined by 2-way ANOVA. receptor at the cell surface (Figure $5 \mathrm{~B}$ ) (23). Using the receptor-HIT configuration $(24,25)$, treatment with Ang II also induced a BRET signal in cells coexpressing RAGE-Rluc 8 and $\beta$-arrestin2-Venus in the presence of the untagged $\mathrm{AT}_{1}$ receptor, again consistent with this interaction occurring at the cell surface. Notably, we observed no BRET following activation of the CCR4 chemokine receptor by CCL22 (Figure 5C). This was despite the fact that CCR4 was still able to activate $\mathrm{G}$ protein (Figure $5 \mathrm{D}$ ) and trigger the recruitment 
of $\beta$-arrestin2-Venus following its activation by its cognate ligand (Figure 5B). As further evidence of the functional interaction between RAGE and the $\mathrm{AT}_{1}$ receptor, we found that RAGE-Rluc8 cellular trafficking was induced upon activation of the $\mathrm{AT}_{1}$ receptor (Figure $5 \mathrm{E}$ ), as shown by its proximity to Venus-tagged markers of specific cellular compartments (26).

An Ang II-induced BRET signal was still observed between $\mathrm{N}$-terminal mCherry-tagged RAGE ${ }_{338-361}$ (the transmembrane and juxtamembrane domains only) and an extracellular luciferasetagged $\mathrm{AT}_{1}$ receptor (Nluc-AT ${ }_{1}$ ) (Figure $5 \mathrm{~F}$ ), with the intracellular luciferase-tagged $\mathrm{AT}_{1}$ receptor $\left(\mathrm{AT}_{1}-\mathrm{Nluc}\right)$ serving as a negative control for this experiment. Moreover, overexpression of unlabeled RAGE ${ }_{338-361}$ inhibited the BRET between full-length RAGE and the $\mathrm{AT}_{1}$ receptor (Figure $5 \mathrm{G}$ ). By contrast, $\mathrm{RAGE}_{362-404}$ had no effect on the BRET between RAGE-Venus and AT -Nluc (Figure $5 \mathrm{H})$. To elucidate the functional involvement of the transmembrane domain of RAGE in Ang II-mediated signaling, RAGE ${ }_{338-361}$ was overexpressed in HMEC1. This resulted in inhibition of the Ang II-induced ICAM-1 expression mediated via endogenous RAGE, which was able to be rescued by RAGE $_{362-404}$ (Figure 5I). This suggests that, while the cytosolic portion of RAGE is necessary for mediating downstream Ang II-induced proinflammatory signaling, the transmembrane domain of RAGE may also be involved in a separate interaction with the $\mathrm{AT}_{1}$ receptor.

Modulation of RAGE transactivation with RAGE oligopeptides. Although transfection with S391A-RAGE ${ }_{362-404}$ or RAGE 370-390 constructs did not have the capacity to be transactivated in $\mathrm{AT}_{1}-\mathrm{CHO}$ cells, transfection of HMEC1 with these constructs prevented the induction of ICAM-1 and $M C P-1$ expression by Ang II via endogenous RAGE in HMEC1 (Figure 6A). To validate the therapeutic potential of directly modulating RAGE ligand-independent signaling via transactivation of RAGE, we generated oligopeptides comprising the C-terminal of RAGE tagged with mCherry fluorescent protein and an HIV-TAT motif to facilitate cellular penetration. Treatment of $\mathrm{AT}_{1}-\mathrm{CHO}$ cells with mCherry-RAGE ${ }_{362-404}(0.4$ $\mathrm{ng} / \mathrm{ml}$ ) was able to induce de novo proinflammatory responsiveness to Ang II (Figure 6B) in the absence of RAGE gene expression. Similarly, in PMAECs from Ager-KO mice, we observed that treatment with mCherry-RAGE ${ }_{362-404}$ was able to restore proinflammatory responsiveness to Ang II (Figure 6C). Treatment with mCherry-RAGE ${ }_{362-404}$ was also able to rescue the inhibition of signaling achieved following treatment with siRNA targeting Ager, Diaph1, or PKC $\zeta$ in HMEC1 (Supplemental Figure 2E and G and Supplemental Figure 5C).

In contrast to the above findings, treatment of RAGEreplete PMAECs with mCherry-S391A-RAGE ${ }_{362-404}$ inhibited the Ang II-mediated induction of NF- $\mathrm{KB}$-dependent genes (Figure 6D). Likewise, mCherry-S391A-RAGE ${ }_{362-404}$ inhibited the Ang IImediated induction of ICAM-1 expression in HMEC1 (Figure 6E) and HAECs (Supplemental Figure 5D), which was comparable to that achieved with the $\mathrm{AT}_{1}$ receptor antagonist irbesartan. We found that pretreatment with mCherry-S391A-RAGE ${ }_{362-404}$ also inhibited Ang II-induced signaling in $\mathrm{AT}_{1}-\mathrm{CHO}$ cells also overexpressing full-length RAGE or $\mathrm{RAGE}_{362-404}$, but failed to inhibit signaling mediated by shorter RAGE constructs (Supplemental Figure 5E). We observed that mCherry-S391A-RAGE ${ }_{362-404}$ also inhibited Ang II-induced signaling in $\mathrm{AT}_{1}-\mathrm{CHO}$ cells expressing
S391Q-cRAGE, a construct devoid of targets for phosphorylation (Supplemental Figure 5F). Pretreatment of $\mathrm{AT}_{1}-\mathrm{CHO}$ cells with mCherry-RAGE ${ }_{362-404}$ did not prevent the inhibitory effects of mCherry-S391A-RAGE ${ }_{362-404}$ (Supplemental Figure 6A). Moreover, the inhibition of Ang II-dependent signaling achieved by S391A-RAGE ${ }_{362-404}$ was not reversed by mCherry-RAGE ${ }_{362-404}$, even at 1000-fold excess (Supplemental Figure 6B). mCherryS391A-RAGE ${ }_{362-404}$ also inhibited RAGE ligand-dependent signaling in PMAECs (Supplemental Figure 6C), but had no effect on ICAM-1 expression induced by TNF- $\alpha$ (Supplemental Figure 6D).

Targeting RAGE-AT receptor interaction in vivo. Pretreatment of whole aortae from Apoe-KO mice with mCherry-S391A-RAGE ${ }_{362-404}$ $(8 \mu \mathrm{g} / \mathrm{ml})$ ex vivo prevented the induction of ICAM-1 expression (Figure 6F) and other key proinflammatory genes (Supplemental Figure $6, \mathrm{E}$ and F) following exposure to Ang II (1 $\mu \mathrm{M}$ for $4 \mathrm{~h})$. In contrast, pretreatment of aortae from Ager/Apoe-DKO mice with mCherry-RAGE ${ }_{362-404}$ restored proinflammatory responsiveness to Ang II (Figure 6F and Supplemental Figure 6, E and F).

In vivo treatment of Ace2/Apoe-DKO mice with mCherryS391A-RAGE ${ }_{362-404}(10 \mu \mathrm{g} / \mathrm{kg}$ i.p. every other day for 10 weeks) also attenuated Ang II-dependent atherosclerosis (Figure 6G), without affecting blood pressure control (Supplemental Table 1). In contrast to this, we observed that treatment with mCherryRAGE $_{362-404}$ reversed the protection afforded by genetic RAGE deletion in Ager/Ace2/Apoe-TKO mice (Figure 6G) and modestly increased atherosclerosis in Apoe-KO mice. Finally, we found that 10 weeks of treatment with mCherry-S391A-RAGE ${ }_{362-404}$ also reduced plaque accumulation in diabetic Apoe-KO mice (Figure $6 \mathrm{H})$, a model associated with RAAS activation, without lowering blood pressure (Supplemental Table 1). Treatment with mCherryRAGE $_{362-404}$ also reversed the protection afforded by genetic RAGE deletion and restored diabetes-accelerated atherogenesis in Ager/Apoe-DKO mice (Figure 6H). However, we observed that treatment with mCherry-RAGE ${ }_{362-404}$ also increased plaque accumulation in RAGE-replete diabetic Apoe-KO mice.

\section{Discussion}

Activation of the $\mathrm{AT}_{1}$ receptor by Ang II leads to the induction of $\mathrm{NF}-\kappa \mathrm{B}(2,3)$ and subsequent inflammation through pathways distinct from those mediating classical Gq-induced signaling $(8,9)$. Here, we show that RAGE ligand-independent transactivation of RAGE following activation of the $\mathrm{AT}_{1}$ receptor was a key determinant of this division. Consequently, many of the proinflammatory changes induced following $\mathrm{AT}_{1}$ receptor activation were attenuated when RAGE was deleted or when RAGE transactivation was inhibited. Also, the proinflammatory effects of $\mathrm{AT}_{1}$ receptor activation were enhanced in the presence of RAGE. In contrast, we observed that $\mathrm{AT}_{1}$ receptor-dependent signaling via classical Gq pathways was unaffected by RAGE expression and that RAGE deletion had no deleterious effect on vascular homeostasis in the setting of salt deficiency or blood pressure responsiveness to Ang II.

Nonspecific crosstalk between the AGE/RAGE axis and the RAAS has long been known, and this crosstalk establishes a vicious cycle for injury and dysfunction (27-34). For example, RAGE ligand-dependent activation of RAGE induces molecular pathways in common with RAAS signaling, including tyrosine phosphorylation of JAK2 and activation of PKC, NF- $\mathrm{BB}$, and 

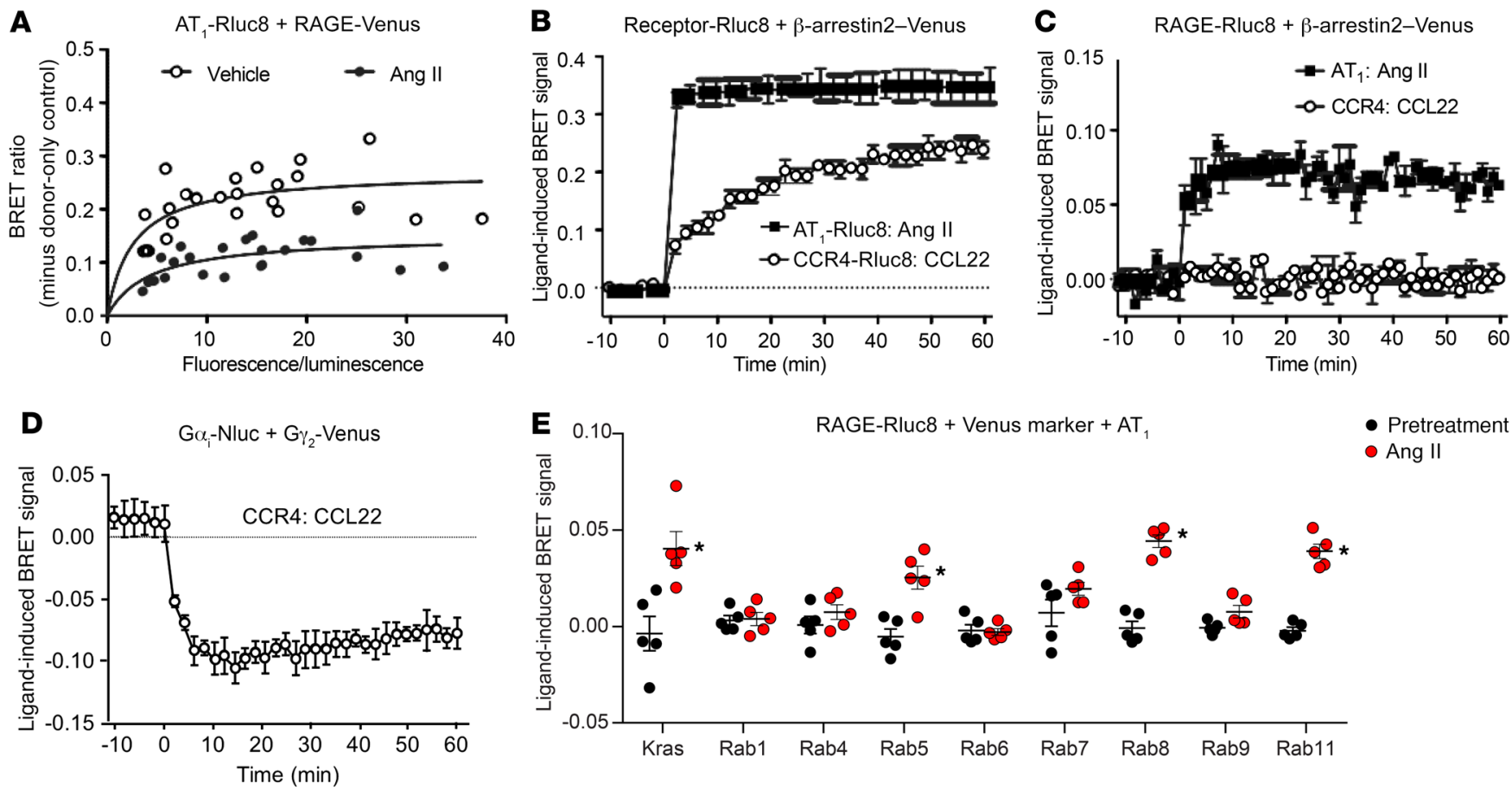

- Pretreatment - Ang II
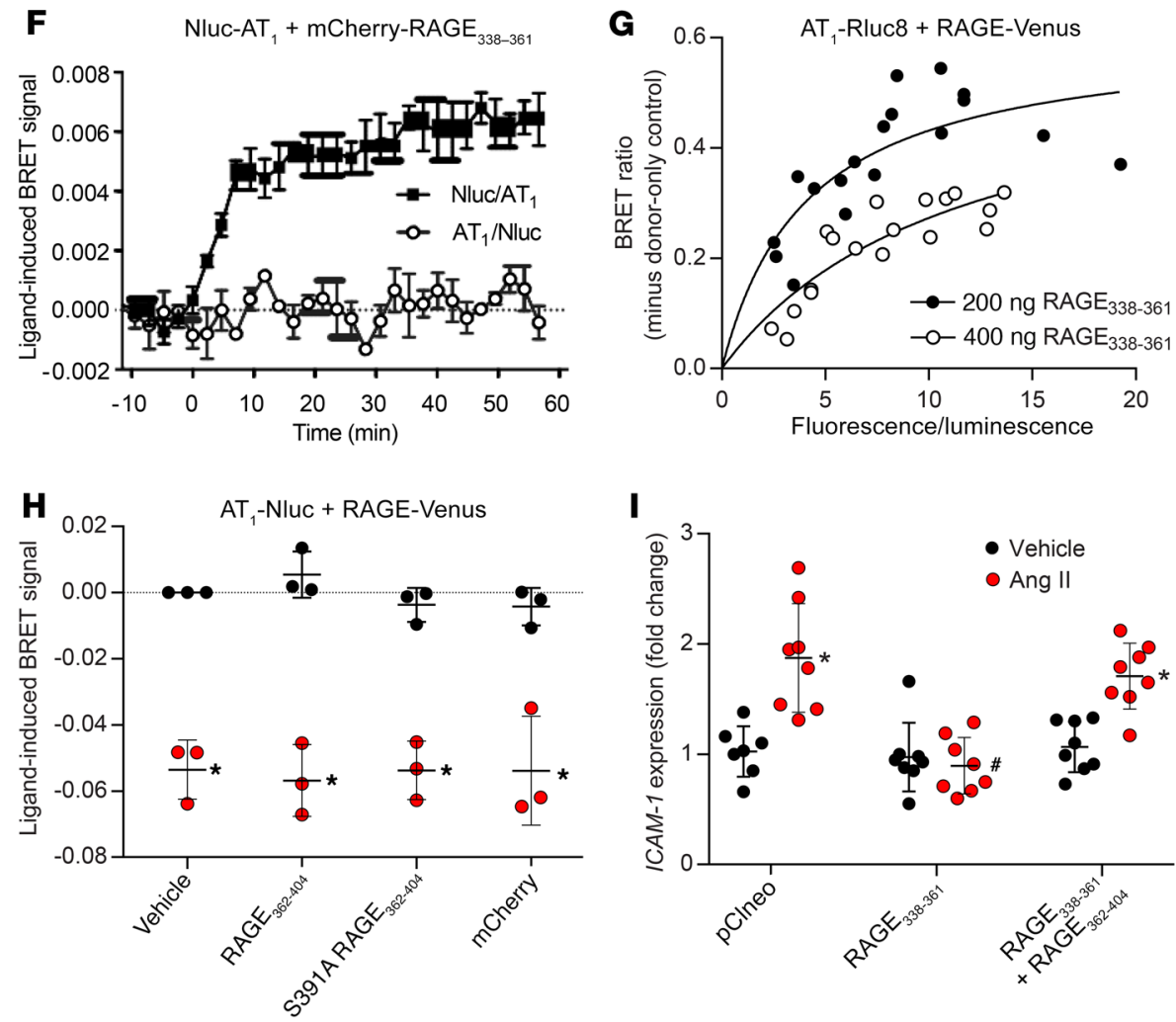

Figure 5. The AT, receptor and RAGE form a functional heteromeric complex. (A) BRET saturation curves with AT - Rluc8 and RAGE-Venus generated 60 minutes after the addition of Ang II or vehicle. (B) Ang II-induced recruitment of $\beta$-arrestin2-Venus to AT-Rluc8 and CCL22-induced recruitment of $\beta$-arrestin2-Venus to CCR4-Rluc8 as a control. (C) Ang II-induced recruitment of $\beta$-arrestin2-Venus proximal to RAGE-Rluc8 only in the presence of AT, following exposure to Ang II, and not in the presence of CCR4 following exposure to CCL22. (D) CCL22-induced BRET signal was observed when Gai-Nluc and $\mathrm{G} \gamma 2$-Venus were coexpressed in the presence of untagged CCR4, serving as an additional control. (E) Ang II-induced trafficking of RACE-Rluc8 in the presence of $A T_{1}$ using markers of specific subcellular compartments. (F) BRET between mCherry-RAGE ${ }_{338-361}$ and Nluc-AT, was increased with Ang II. (C) BRET between AT - -Rluc8 and RAGE-Venus was inhibited by mCherry-RAGE ${ }_{338-361}$. $(\mathrm{H})$ BRET between AT - Nluc and RAGE-Venus was reduced by Ang II but not affected by mCherry-RAGE ${ }_{362-404^{*}}$ (I) Ang II-mediated proinflammatory signaling in HMEC1 inhibited by RAGE 338-361 was rescued by mCherry-RACE ${ }_{362-404}$ Data are presented as the mean \pm SD. $n=3(\mathbf{B}-\mathbf{D}$ and $\mathbf{H}), n=5(\mathbf{E}$ and $\mathbf{F}), n=6-8(\mathbf{I})$, or data were combined from 3 independent experiments $(\mathbf{A}$ and $\mathbf{G})$. ${ }^{*} P<0.05$ versus vehicle or pretreatment or 0 minutes; ${ }^{*} P<0.05$ versus other treated conditions. $P$ values were determined by 2 -way ANOVA. 

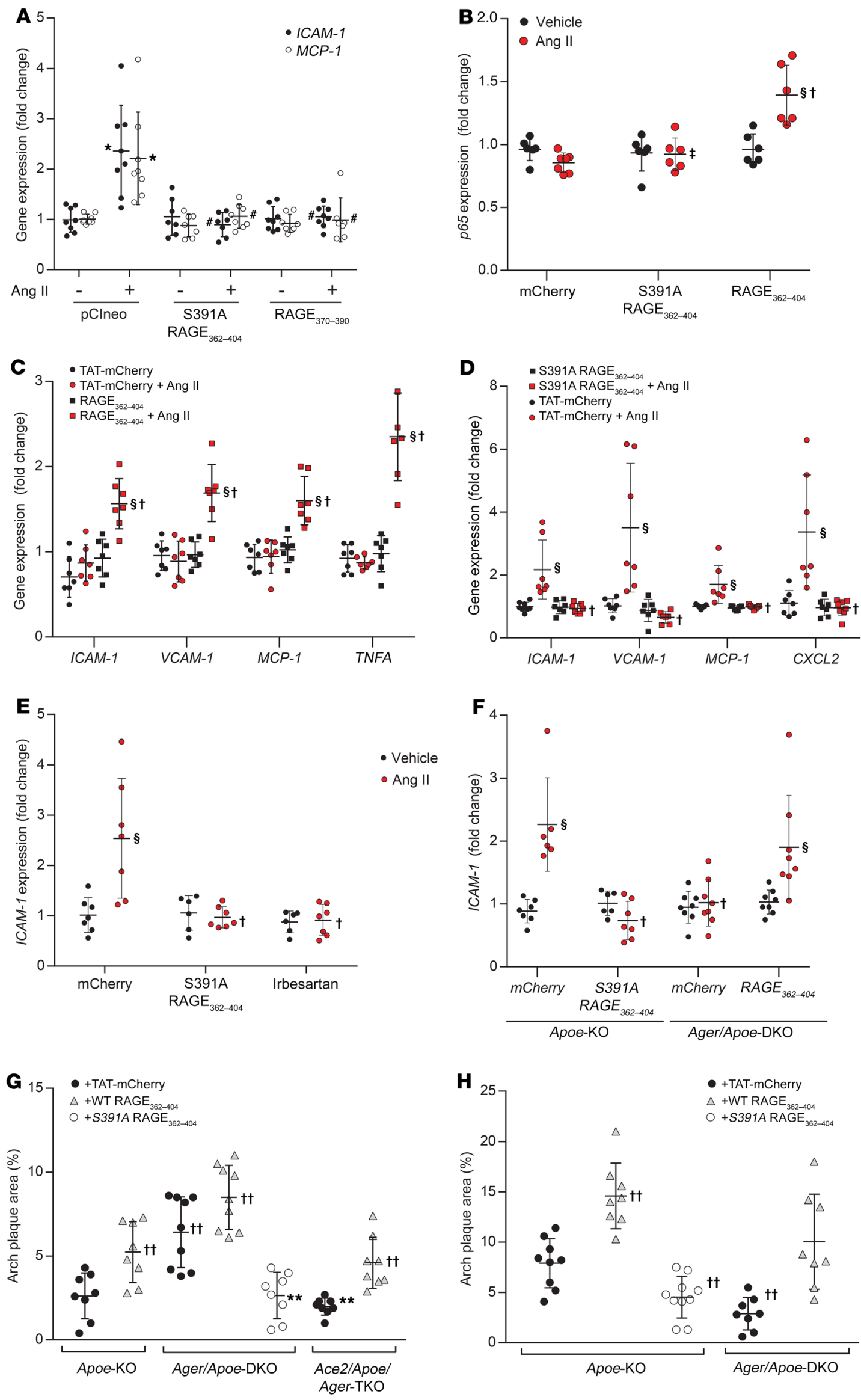
Figure 6. Effects of treatment with mCherry-RAGE ${ }_{362-404}$ oligopeptides on proinflammatory signaling following exposure to Ang II in vitro, ex vivo, and in vivo. Treatment with Ang II was at a dose of $1 \mu \mathrm{M}$.(A) Induction of ICAM-1 and MCP-1 expression in HMEC1 in the presence or absence of plasmids expressing truncated RAGE constructs. (B) Induction of $p 65$ expression in $\mathrm{AT}_{1}-\mathrm{CHO}$ cells treated with mCherry-RACE $\mathrm{B}_{362-404}$ or mCherry-S391A-RAGE ${ }_{362-404}(0.4 \mathrm{ng} / \mathrm{ml})$. (C) Expression of proinflammatory genes in PMAECs from Ager-KO mice in the presence or absence of mCherry-RACE ${ }_{362-404}(0.4 \mathrm{ng} / \mathrm{ml})$. (D) Expression of proinflammatory genes in (RAGE-replete) PMAECs induced by Ang II in the presence or absence of mCherry-S391A-RAGE ${ }_{362-404}(0.4 \mathrm{ng} / \mathrm{ml})$. (E) Induction of ICAM-1 expression in PMAECs in the presence or absence of mCherry-S391A-RAGE ${ }_{362-404}(0.4$ $\mathrm{ng} / \mathrm{ml})$ or irbesartan $(1 \mu \mathrm{M})$. (F) Induction of ICAM-1 expression in aortae from Apoe-KO and Ager/Apoe-DKO mice in the presence or absence of mCherry-RAGE ${ }_{362-404}$ or mCherry-S391A-RACE ${ }_{362-404}(8 \mathrm{ng} / \mathrm{ml})$. (C) Plaque area in aortae from Apoe-KO, Ace2/Apoe-DKO, and Ager/Ace2/Apoe-TKO mice in the presence or absence of mCherry-RAGE ${ }_{362-404}$ or mCherry-S391ARACE $_{362-404}(10 \mu \mathrm{g} / \mathrm{kg}$ i.p. every other day). (H) Plaque area in aortae from diabetic Apoe-KO and Ager/Apoe-DKO mice in the presence or absence of mCherry-RAGE ${ }_{362-404}$ or mCherry-S391A-RAGE ${ }_{362-404}(10 \mu \mathrm{g} / \mathrm{kg}$ i.p. every other day). Data are presented as the mean $\pm S D$. $n=6-8$ group. ${ }^{*} P<0.05$ versus $p C$ Ineo plus vehicle; ${ }^{\#} P<0.05$ versus $p C$ Ineo plus Ang II; ${ }^{\circledR} P<0.05$ versus $\mathrm{mCherry}$ plus vehicle; ${ }^{\dagger} P<0.05$ versus mCherry plus Ang $\mathrm{II} ;{ }^{\ddagger} P<$ 0.05 versus mCherry-RAGE ${ }_{362-404}$ plus Ang II; ${ }^{* *} P<0.05$ versus Ace2/ Apoe-DKO plus mCherry; ${ }^{+\dagger} P<0.05$ versus Apoe plus TAT-mCherry. $P$ values were determined by 2-way ANOVA.

NADPH oxidase (27-29). Blockade of the RAAS has also been shown to attenuate the effects of AGE-induced activation of RAGE (30-33). Likewise, dicarbonyl scavengers, which reduce the formation of RAGE-activating AGEs, attenuate Ang II-dependent injury (34). In certain cell types, Ang II-mediated signaling is known to transactivate growth receptor tyrosine kinases with a proposed mechanism being metalloprotease-mediated shedding of membrane-tethered ligands (21), and RAAS activation certainly increases RAGE ligands. We had initially speculated that this kind of extracellular pathway may exist for RAGE transactivation. However, we found that a neutralizing antibody targeting the ligand-binding domain of RAGE or a soluble decoy receptor had no effect on Ang II-mediated signaling in vitro. Moreover, deletion of the extracellular domain of RAGE did not modify the ability of N-truncated RAGE constructs to transduce the proinflammatory effects of Ang II. Indeed, we observed that treatment with $\mathrm{RAGE}_{362-404}$ fully restored the Ang II-dependent induction of inflammatory mediators in vitro and ex vivo and atherogenesis in vivo in the absence of full-length RAGE. Nonetheless, the (RAGE-dependent) liberation of RAGE ligands following activation of the $\mathrm{AT}_{1}$ receptor may play a latent role in sustaining or enhancing proinflammatory signaling via RAGE, including the autoinduction of RAGE expression (13). This may partly explain why soluble RAGE, comprising only the ligand-binding domain of RAGE, has some vasculoprotective effects in vivo (16), although other actions including the antagonism of ligand-mediated activation of TLRs may also contribute to this protection.

Here, we provide evidence that RAGE and the $\mathrm{AT}_{1}$ receptor form a heteromeric complex at the plasma membrane, as demonstrated by the generation of a strong and saturable BRET signal between $\mathrm{AT}_{1}$-Rluc8 and RAGE-Venus, as well as the receptor-HIT signal with RAGE-Rluc8 observed when $\beta$-arrestin2-Venus was recruited to the $\mathrm{AT}_{1}$ receptor following Ang II-induced activation.
We revealed additional evidence for the functional interaction between the 2 receptors with the BRET trafficking assay, in which coexpression and activation of the $\mathrm{AT}_{1}$ receptor resulted in trafficking of RAGE-Rluc8 through various cellular compartments, including early endosomes (Rab5), recycling endosomes (Rab11), and forward trafficking from the trans-Golgi network to the plasma membrane (Rab8), consistent with the strong expression of RAGE on the plasma membrane proximal to the Kras marker. This interaction between RAGE and the $\mathrm{AT}_{1}$ receptor appears to be dependent on the transmembrane domain of RAGE, such that BRET was demonstrated between Nluc-AT ${ }_{1}$ and mCherry-RAGE ${ }_{338-361}$, and unlabeled RAGE ${ }_{338-361}$ inhibited the BRET between full-length RAGE and the $\mathrm{AT}_{1}$ receptor. In contrast, the extracellular and cytosolic domains of RAGE were unable to modulate the BRET signal between the $\mathrm{AT}_{1}$ receptor and full-length RAGE. Interestingly, we observed that overexpression of transmembrane $\mathrm{RAGE}_{338-361}$ also inhibited Ang II-dependent signaling by endogenous RAGE in HMEC1, although this inhibition was overcome by cytosolic RAGE $_{362-404}$. This indicates that, when free from the constraints of being membrane bound, the cytosolic domain of RAGE independently retains the capacity to be transactivated by the $\mathrm{AT}_{1}$ receptor and associated proteins. However, while the 2 domains could function separately, they were mutually dependent for signaling via endogenous membrane-bound RAGE.

Previous studies have suggested that PKC $\zeta$-mediated phosphorylation of S391 on RAGE is a key step in RAGE activation (35). Among the 4 potential phosphorylation sites in human RAGE, S391 is conserved among humans, mice, horses, rabbits, dogs, and cats (Supplemental Table 2). However, S391Q-RAGE (as found in camels) and S391P-RAGE (as found in cattle, pigs, sheep, and goats) are both able to be activated by Ang II or S100A8/9, even following the additional mutation of the other possible phosphorylation targets in the cytosolic tail, demonstrating that phosphorylation of RAGE is not essential for its activation. PKC $\zeta$ is known to associate with the cytosolic domain of RAGE and was thought to mediate ligand-mediated phosphorylation of RAGE (35). PKC $\zeta$ certainly appeared to be critical for transactivation of full-length RAGE by the $\mathrm{AT}_{1}$ receptor, as selective inhibition of $\mathrm{PKC} \zeta$ was able to prevent activation of NF- $\kappa \mathrm{B}$ following exposure to Ang II. However, inhibition of PKC $\zeta$ also prevented activation of S391QcRAGE by Ang II or S100A8/9, demonstrating that PKC $\zeta$ was functioning independently of RAGE phosphorylation. In contrast, signaling via RAGE $_{362-404}$ was not prevented following silencing of $\mathrm{PKC} \zeta$, suggesting that $\mathrm{PKC} \zeta$ plays no direct role in transactivation of the cytosolic tail of RAGE.

The interaction of RAGE with Diaph1 is also thought to be important for ligand-dependent signaling (22, 36), possibly because it facilitates the recruitment and Ras-dependent activation of PKCל, with which Diaph1 also binds (37). In our experiments, silencing of Diaph1 expression also inhibited Ang II-induced proinflammatory signaling via full-length RAGE. However, the role of Diaph1 in RAGE ligand-dependent activation also appears to be different from its role in RAGE ligandindependent transactivation. Specifically, mutation of the charged patch (R366A-Q367A-RAGE) prevents signaling by RAGE ligands, as previously described $(22,36)$, but has no effect on Ang II-induced RAGE transactivation. Indeed, the activity of 
full-length RAGE is retained by $\mathrm{RAGE}_{370-404}$, despite the absence of the charged patch in this construct. It may be that $\mathrm{AT}_{1}$ receptor activation is able to independently induce the activation of Diaph1. Diaph1 is a known downstream effector of Rho-GTPases (38), and Rho-A, a key product of $\mathrm{AT}_{1}$ receptor activation, is known to trigger the activation of Diaph1, even in the presence of the R366A-Q367A RAGE mutation (39). However, we found that RAGE $_{362-404}$ was not inhibited following the silencing of Diaph1 expression, similar to what we observed following the silencing of PKC $\zeta$ (Figure 4F). This suggests that the role of RAGE and $\mathrm{PKC} \zeta$ in the propagation of proinflammatory signaling may also involve the transmembrane domain, possibly to regulate membrane localization, similar to what was reported for translocation of the RAGE-binding partner Src (40).

Only some cells (e.g., endothelial cells, leukocytes) express RAGE under basal conditions, meaning that the transactivation pathway described in this report is basally limited to these cell types. Most other cells do not express RAGE, including healthy smooth muscle cells (10) and HepG2, a cell type in which Ang II notably fails to induce canonical NF- $\mathrm{BB}$ signaling (41). In addition to the fact that RAGE expression had no effect on the classical Gq signaling induced following $\mathrm{AT}_{1}$ receptor activation, this limited basal distribution of RAGE may partly explain why RAGE deletion or inhibition did not affect blood pressure, natriuresis, or other homeostatic functions of the systemic RAAS. However, following injury, inflammation, or hypoxia, the de novo or enhanced expression of RAGE may provide a conduit for the proinflammatory effects of local RAAS activation. In such states, the blood pressure-independent benefits of RAAS blockade may be partly attributable to the inhibition of RAGE transactivation and associated RAGE autoinduction. However, the efficacy of systemic RAAS blockade is often constrained by the limits of blood pressure lowering (e.g., following a myocardial infarction or in severe heart failure) and feedback "escape" activation of the RAAS (42). One potential advantage of targeting RAGE transactivation is that these key limitations of systemic RAAS blockade do not apply.

As a proof of principle, we designed cell-penetrating RAGE oligopeptides that differed by only 1 amino acid and had the capacity to independently mediate $\left(\mathrm{RAGE}_{362-404}\right)$ or selectively inhibit (S391A-RAGE ${ }_{362-404}$ ) Ang II-induced activation of proinflammatory pathways contributing to RAAS-dependent atherosclerosis. As an inhibitory strategy, the blockade achieved was as effective as an AT receptor antagonist in vitro, but without adverse effects on hemostasis in vivo. Inhibition with S391A-RAGE ${ }_{362-404}$ was achieved at low concentrations and not overcome by WT RAGE ${ }_{362-404}$, even at 1000-fold excess. Taken together, these data suggest that conformational changes induced following substitution or deletion of S391 make it more adept at (or less constrained in) interacting with key elements in the RAGE transactivation pathway. Additional mutation or deletion of the Diaph1-binding domain may further reduce structural constraints and improve binding efficiency, explaining how $\mathrm{RAGE}_{370-404}$ could overcome the inhibition conferred by S391A-RAGE ${ }_{362-404}$ over full-length RAGE.

Even though ligand binding to the ectodomain has historically been considered essential for the functions of RAGE (43), the RAGE ligand-independent transactivation pathway described in our study appears to be a dominant mechanism inducing its activation and downstream effector signaling (39). Consistent with this hypothesis, we found that treatment with RAGE $_{362-404}$ fully restored the susceptibility of Ager-KO mice to atherogenesis, without restoring the signaling pathways mediated by elevated RAGE ligands, such as AGEs and S100 proteins. This suggests that the relative vasculoprotection observed in Ager-KO mice was largely mediated through inhibition of RAGE transactivation, highlighting its pathophysiological importance and providing a clear rationale for its inhibition. We hypothesize that a mechanistic understanding of the $\mathrm{AT}_{1}$-RAGE heteromer will lead to the development of novel therapeutics that are able to specifically target the adverse effects of RAGE transactivation, without compromising blood pressure regulation or inducing feedback "escape" that limits conventional RAAS blockade. These inhibitors may be applicable across a broad range of conditions in which RAGE has been implicated, including atherosclerosis (14), neurodegenerative diseases $(11,44)$, malignancy $(12)$, diabetic complications $(13,14)$, and other important inflammatory conditions.

\section{Methods}

\section{Animal models}

To investigate the impact of RAGE in RAAS-dependent pathology in vivo, we used the Apoe-KO mouse, which is a model associated with enhanced atherosclerotic plaque accumulation following RAAS activation and vasculoprotection with RAAS blockade $(4,5,7,19)$. However, the unusual dyslipidemia, the absence of spontaneous plaque rupture, and the independent proinflammatory effects of Apoe deletion potentially limit the applicability of this mouse model to human disease. C57BL/6J mice, Ager-KO mice, Apoe-KO mice, Ager/Apoe-DKO, Ace2/Apoe-DKO, and Ager/Ace2/Apoe-TKO mice (all backcrossed $>10$ generations) were sourced and generated in house, as previously described $(14,43,45)$. All mice were bred on a C57BL/6J background. For the experimental studies, male mice aged 6-8 weeks and weighing between 20 and $25 \mathrm{~g}$ were used, with at least 8 animals per group.

Ang II infusion. Apoe-KO and Ager/Apoe-DKO mice were randomized to receive Ang II ( $1 \mu \mathrm{g} / \mathrm{kg} / \mathrm{min}$; MilliporeSigma) or vehicle via s.c. infusion for 4 weeks using an osmotic minipump (model 2004, ALZET, BioScientifica) placed into the s.c. space along the dorsal midline of each mouse, under general anesthesia.

Low-sodium diet. Exposure to a low-sodium diet (0.05\% sodium) is an experimental model of physiological RAAS activation (46). In this study, Apoe-KO and Ager/Apoe-DKO mice were randomized to receive an isocaloric diet containing $6 \%$ fat and either low sodium $(0.05 \%)$ or normal sodium (0.3\%) amounts (Specialty Feeds) for 1 week or 6 weeks.

After 1 week of study, Apoe-KO and Ager/Apoe-DKO animals were humanely euthanized and the aortae were isolated, cleaned of fat, and mounted in a vessel chamber primed with Krebs buffer and maintained at a physiological $\mathrm{pH}$ by infusing carbogen gas $\left(95 \% \mathrm{O}_{2}\right.$, $5 \% \mathrm{CO}_{2}$ ) through the buffer at $37^{\circ} \mathrm{C}(19)$. Vessels ( $n=6 /$ group) were pretreated with Ang II $(1 \mu \mathrm{M})$ or vehicle for 4 hours at $37^{\circ} \mathrm{C}$. Whole blood labeled with DilC18 (1:1000) was then perfused through the aorta at $0.12 \mathrm{ml} /$ minute. Images and videos of vessel wall-cell interactions were acquired using a fluorescence microscope (Zeiss Discovery. V20), coupled to a digital camera (Hamamatsu ORCA-ER) and analyzed with AxioVison software. 
A second group of mice was studied for 6 weeks, after which time the mice were placed into individual metabolic cages (Iffa Credo) for 24 hours, and their weight, water and food (sodium) intake, and urine output were documented. The urinary sodium concentration was measured with a COBAS-INTEGRA 400 Autoanalyzer (Roche Diagnostics) using an ion-sensitive electrode, and the result was adjusted for urinary output ( $\mu \mathrm{mol} / \mathrm{day}$ ).

Ace2-KO mice. In these studies, male Ace2/Apoe-DKO and Ager/ Ace2/Apoe-TKO mice were followed out to 18 weeks of age, a time point associated with increased plaque accumulation in DKO mice (19). In a second set of experiments, the mice, from 6 to 8 weeks of age, were further randomized to receive the mCherry-S391A-RAGE ${ }_{362-404}$ oligopeptide $\left(10 \mu \mathrm{g} / \mathrm{kg}\right.$ i.p. every other day), the mCherry-RAGE ${ }_{362-404}$ oligopeptide (10 $\mu \mathrm{g} / \mathrm{kg}$ i.p. every other day), or mCherry alone (control; $10 \mu \mathrm{g} / \mathrm{kg}$ i.p. every other day).

Streptozotocin diabetes. Apoe-KO mice and Ager/Apoe-DKO were randomly allocated to receive streptozotocin $(55 \mathrm{mg} / \mathrm{kg}$, Sigma Chemical Co.) or buffer (sodium citrate buffer, $\mathrm{pH}$ 4.5) delivered i.p. in 5 consecutive daily doses, followed by mCherry-S391A-RAGE ${ }_{362-404}$ oligopeptide $\left(10 \mu \mathrm{g} / \mathrm{kg}\right.$ i.p. every other day), the mCherry-RAGE ${ }_{362-404}$ oligopeptide (10 $\mu \mathrm{g} / \mathrm{kg}$ i.p. every other day), or mCherry alone (control; $10 \mu \mathrm{g} / \mathrm{kg}$ i.p. every other day) for 10 weeks. All diabetic mice achieved an $\mathrm{HbA1c}$ of more than $8 \%$ (median $11.2 \%$ ) after 10 weeks, with no significant differences between treatment groups (data not shown).

Paigen diet. To exclude the nonspecific antiatherogenic actions of RAGE deletion, Apoe-KO and Ager/Apoe-DKO mice were fed a high-fat (Paigen) diet composed of $18.5 \%$ fat, $0.9 \%$ cholesterol, and $0.5 \%$ cholate for 6 weeks (20). This is considered a lipid-dependent model of atherosclerosis and is not inhibited by conventional RAAS blockade, unlike the other models detailed above.

General assessments. Systolic blood pressure was measured by tailcuff plethysmography in conscious, prewarmed mice using a computerized, noninvasive tail-cuff system (Kent Scientific). Mice from the low-sodium diet study underwent an additional measurement of plasma renin activity and serum aldosterone levels (radioimmunoassay) (ProSearch International) as well as circulating soluble MCP-1 and ICAM-1 protein levels (R\&D Biosystems). In Ace2-KO mice, Ang II levels were specifically measured by radioimmunoassay (ProSearch International).

Plaque area quantitation. After the animals were humanely euthanized, the aortic arches were removed, cleaned of excess fat, placed in $10 \%$ neutral buffered formalin, and then stained with Sudan IV Herxheimer's solution ( $0.5 \% \mathrm{w} / \mathrm{vol}$; Gurr, BDH Ltd.). The aortae were then dissected longitudinally and pinned flat onto a wax pad. Plaque accumulation across the aortic arch and total aortic surface were quantitated as the percentage of area stained red, as previously described $(14,43,45)$.

Quantitative real-time reverse transcription PCR. Aortae were snapfrozen and stored at $-80^{\circ} \mathrm{C}$ in TRIzol (Thermo Fisher Scientific). Next, RNA was extracted and cDNA generated using the TRIzol method (19). Gene expression of key proatherogenic mediators was estimated by quantitative real-time reverse transcription PCR (RT-PCR), performed using the TaqMan system (Thermo Fisher Scientific) on the basis of real-time detection of accumulated fluorescence (ABI Prism 7700, PerkinElmer) (14). Gene expression was normalized to $18 \mathrm{~S}$ mRNA and reported as the fold change compared with the expression level in the untreated control mice, which was assigned an arbitrary value of 1.0.

Ex vivo studies. Aortae were isolated from Apoe-KO and Ager/ Apoe-DKO mice, divided, mounted onto a vessel chamber in Krebs buffer, and maintained at a physiological $\mathrm{pH}$ level by infusing carbogen gas $\left(95 \% \mathrm{O}_{2}, 5 \% \mathrm{CO}_{2}\right)$ through the buffer at $37^{\circ} \mathrm{C}$. The vessels ( $n=6$ /group) were then treated with Ang II ( $1 \mu \mathrm{M}$ for $4 \mathrm{~h}$ ), after which whole blood labeled with Vybrant DiI (1:1000, Lonza) for the fluorescence labeling of leukocytes was then perfused through the aorta at $0.12 \mathrm{ml} /$ minute. Images and videos of vessel wall-cell interactions were observed using a fluorescence microscope (Zeiss Discovery. V20), coupled to a digital camera (Hamamatsu ORCA-ER) and analyzed with AxioVison software.

In a second set of experiments, aortae isolated from Apoe-KO and Ager/Apoe-DKO mice were randomized to receive pretreatment with mCherry (control), mCherry-RAGE ${ }_{362-404}$, or mCherry-S391ARAGE $_{362-404}$ oligopeptide (all $8 \mu \mathrm{g} / \mathrm{ml}$ ). Thirty minutes later, the aortae were exposed to Ang II ( $1 \mu \mathrm{M}$ for $4 \mathrm{~h})$, after which changes in the expression of key NF- $\mathrm{kB}$-dependent proinflammatory genes were assessed using real-time RT-PCR.

\section{In vitro studies}

PMAECs. To assess the impact of RAGE deficiency on NF- $\mathrm{KB}$-dependent proinflammatory signaling via the $\mathrm{AT}_{1}$ receptor, PMAECs were isolated from the aortae of (WT) C57BL/6J mice and Ager-KO mice, as previously described (19), and cultured in DMEM/F-12 endothelial cell growth supplement (ECGS) media. Cultured PMAECs were then exposed to Ang II ( $1 \mu \mathrm{M}$ for 2 hours), the RAGE ligand S100A8/ A9 $(2 \mu \mathrm{g} / \mathrm{ml}$ for $2 \mathrm{~h})$, or vehicle, after which the cells were placed in TRIzol, the mRNA extracted, and the cDNA synthesized. Changes in gene expression of the RelA $p 65 \mathrm{NF}-\kappa \mathrm{B}$ subunit gene, the Egr1 gene, or $\mathrm{NF}-\kappa \mathrm{B}$-activated target genes were estimated by quantitative real-time RT-PCR, as detailed above. To confirm that the $\mathrm{AT}_{1}$ receptor signaling cascade was functional in PMAECs from Ager-KO mice, the induction of $\mathrm{IP}_{1}$, a stable downstream metabolite of $\mathrm{IP}_{3}$, which accumulates in cells following Gq receptor activation, was measured 2 hours after exposure to Ang II or vehicle using the IP-One HTRF (homogeneous time-resolved fluorescence) assay (CisBio Bioassays).

Functional responsiveness to Ang II was further determined in PMAECs using a static adhesion assay. In brief, PMAECs were seeded at 50,000 cells per well into 6-well plates and allowed to grow to $70 \%$ confluency before treatment with $1 \mu \mathrm{M}$ Ang II for 24 hours. Human THP-1 cells were stained using the CellVue Burgundy Fluorescent Cell Labeling Kit (LI-COR), before seeding them onto the endothelial cell monolayers at $3 \times 10^{5}$ viable cells per well, and incubated for 20 minutes at $37^{\circ} \mathrm{C}$. The wells were washed with PBS before fixation of the cells with $4 \%$ formalin in PBS for 30 minutes. The adhesion of cells was quantitated using the ODYSSEY infrared imager (LI-COR).

In separate experiments, PMAECs from C57BL/6J mice were transfected with siRNAs targeting Ager (Ambion, assay ID: s62119; $2 \mathrm{nM}$ ), p65 (RelA) (Ambion, assay ID: s72857; $10 \mathrm{nM}$ ), PKC (Ambion, s71716; $10 \mathrm{nM}$ ), Diaph1 (sense 5'-UACAGAGGAAGCUGAUAUUGAAGCC, antisense 3'-GGCUUCAAU AUCAGCUUCCUCUGUA; Invitrogen, Thermo Fisher Scientific; $10 \mathrm{nM}$ ), or scrambled control 1 (Ambion; 2 $\mathrm{nM}$ or $10 \mathrm{nM}$ ) using Lipofectamine RNAiMAX (Invitrogen, Thermo Fisher Scientific) according to the manufacturer's instructions. The cells were allowed to recover in media containing 10\% FBS for 16 hours prior to treatment with Ang II ( $1 \mu \mathrm{M}$ for 2 hours), S100A8/A9 (2 $\mu \mathrm{g} / \mathrm{ml}$ for $2 \mathrm{~h}$ ), or vehicle. Further experiments were also conducted in the presence and absence of a 1-hour pretreatment with a neutralizing antibody against RAGE (R\&D Systems, monoclonal mouse IgG2B 
clone 176902, catalog MAB11451; $1 \mu \mathrm{g} / \mathrm{ml})$, soluble RAGE $(1 \mu \mathrm{g} / \mathrm{ml})$, or a pseudosubstrate for $\mathrm{PKC} \zeta(5 \mu \mathrm{M}$, Tocris). As a further control, the inhibitory effect of the $\mathrm{AT}_{1}$ receptor antagonist irbesartan $(1 \mu \mathrm{M} ; 30$ min before dosing with Ang II) was also explored.

To quantitate hydrogen peroxide production induced by Ang II in PMAECs, a vessel chamber assay was used to measure peroxide production in real time in PMAECs from Ager-KO and C57BL/6J aortae, induced following exposure to Ang II. PMAECs were grown on collagen-coated coverslips and mounted into a sealed glass chamber when they were $80 \%$ confluent. The chambers were perfused with Krebs buffer with 2,7-dichlorofluorescein (DCFH) (Molecular Probes Europe BV) for 1 hour, with fluorescence readings taken every $10 \mathrm{~min}$ utes to establish baseline values. The cells were then perfused with 1 $\mu \mathrm{M}$ Ang II for 60 minutes, and fluorescence readings were taken every 10 minutes afterward. Fluorescence readings were expressed as the change $(\Delta)$ in fluorescence AU from baseline.

Primary bone marrow-derived macrophages and splenocytes. To examine the impact of Ager deficiency on proinflammatory pathways implicated in the development of atherosclerosis, macrophages were isolated from bone marrow using the inflammasome method (19), and their proinflammatory responsiveness was then determined by changes in gene expression, as assessed by quantitative real-time RT-PCR.

Splenocytes were isolated from Ager-KO and C57BL/6J mice as previously described (47). Proinflammatory responsiveness following exposure to Ang II ( $1 \mu \mathrm{M}$ for $2 \mathrm{~h}$ ) was determined by changes in gene expression of Ccr2, Ifng, and the activation marker Mac1, as assessed by real-time RT-PCR $(19,47)$. Functional responsiveness was estimated by the induction of cellular adhesion between the isolated splenocytes and a monolayer of PMAECs.

Immortalized HMECs. To explore the potential to selectively modulate the transactivation of endogenous RAGE via transfection of human cells, HMECs (HMEC1) (American Type Culture Collection [ATCC]) cultured in MCDB 131 medium (10\% FCS with 10 mM glutamine, EGF, and hydrocortisone) were used. HMEC1 were transfected with AGER constructs using Lipofectamine 2000 (Invitrogen, Thermo Fisher Scientific) according to the manufacturer's instructions. In separate experiments, HMEC1 were also transfected with $2 \mathrm{nM}$ siRNA against AGER (S1167/8), PKCל (S11128), DIAPH1 (S4092/94), or scrambled control 1 (Ambion, Thermo Fisher Scientific) using Lipofectamine RNAiMAX (Invitrogen, Thermo Fisher Scientific) according to the manufacturer's instructions. Cells were allowed to recover in optimum media containing $10 \%$ FBS for 16 hours prior to treatment for 2 hours with $1 \mu \mathrm{M}$ Ang II or $2 \mu \mathrm{g} / \mathrm{ml}$ of the RAGE ligand S100A8/ A9 as a signaling control. HMEC1 were also treated with mCherry (control), mCherry-RAGE ${ }_{362-404}$, or mCherry-S391A-RAGE ${ }_{362-404}$ at a dose of $0.4 \mathrm{ng} / \mathrm{ml}$ for 30 minutes prior to treatment with either Ang II $(1 \mu \mathrm{M})$ or S100A8/A9 $(0.6 \mu \mathrm{g} / \mathrm{ml})$ or vehicle control for 2 hours, after which changes in the expression of key NF- $\kappa \mathrm{B}-$ dependent proinflammatory genes and target genes of the siRNA were assessed by quantitative real-time RT-PCR using the TaqMan system, as detailed above.

Human aortic endothelial cells. To confirm the applicability of inhibition strategies to human tissues, additional experiments were undertaken in primary human aortic endothelial cells (HAECs) (Lonza). HAECs were grown in EGM-2 media (Lonza). Cells were treated with mCherry (control), mCherry-RAGE ${ }_{362-404}$, or mCherryS391A-RAGE ${ }_{362-404}$ at a dose of $0.4 \mathrm{ng} / \mathrm{ml}$ for 30 minutes prior to treatment with either Ang II $(1 \mu \mathrm{M})$ or S100A8/A9 $(0.6 \mu \mathrm{g} / \mathrm{ml})$ for 2 hours.
As a further control, the inhibitory effect of the $\mathrm{AT}_{1}$ receptor antagonist irbesartan $(1 \mu \mathrm{M} ; 30$ minutes before dosing with Ang II) was also explored in HAECs.

Chinese hamster ovary cells. Chinese hamster ovary (CHO) cells (ATCC) were used to explore the signaling interaction between RAGE and the $\mathrm{AT}_{1}$ receptor, as $\mathrm{CHO}$ cells express recombinant proteins well and lack endogenous surface expression of RAGE, $\mathrm{AT}_{1}$ receptors, or other transactivatable elements including EGFR or mineralocorticoid receptor. The pcDNA3.AGTR1-Rluc8 construct (100 ng) (provided by Wally Thomas, University of Queensland, St. Lucia, Brisbane, Australia) was stably transfected into $\mathrm{CHO}$ cells using Lipofectamine 2000 (Thermo Fisher Scientific). AT - - CHO cells were then transiently transfected with RAGE constructs using Lipofectamine 2000 (Invitrogen, Thermo Fisher Scientific). The control for each experiment was transfection with pCIneo or mCherry for full-length RAGE and N-truncated RAGE constructs, respectively. After incubation for 16 hours, the transfected cells were treated with Ang II $(1 \mu \mathrm{M})$, S100A8/A9 $(2 \mu \mathrm{g} /$ $\mathrm{ml}$ ), or vehicle for 2 hours, after which changes in the gene expression of $p 65$ (RelA) and Egr1 were determined by quantitative real-time RT-PCR using the TaqMan system. In some experiments, $\mathrm{AT}_{1}-\mathrm{CHO}$ cells were also pretreated for 30 minutes with mCherry (control), mCherry-RAGE ${ }_{362-404}$, or mCherry-S391A-RAGE ${ }_{362-404}$ at a dose of 0.4 $\mathrm{ng} / \mathrm{ml}$. Further experiments were also conducted in the presence and absence of a 1-hour pretreatment with a neutralizing antibody against RAGE (R\&D Systems; $1 \mu \mathrm{g} / \mathrm{ml}$ ) or soluble RAGE. To directly measure the induction of NF- $\mathrm{\kappa B}$ activity, $\mathrm{AT}_{1}-\mathrm{CHO}$ cells were transfected with $0.4 \mu$ g plasmids encoding NF- $\kappa \mathrm{B}-\mathrm{SEAP}$ or $\beta$-gal using Lipofectamine 2000 (Invitrogen, Thermo Fisher Scientific). After 24 hours, the cells transfected with each plasmid combination were then treated with Ang II, S100A8/A9, or vehicle and incubated for 4 hours before the cell supernatants and cell lysates were collected. The cell supernatants were analyzed using a chemiluminescent secreted alkaline phosphatase (SEAP) Reporter Gene Assay (Roche Applied Science). To control for transfection efficiency, the cell lysates were assayed for $\beta$-gal using a commercially available kit (Promega).

Generation of human Ager and mutant Ager constructs. The Homo sapiens AGER transcript variant 1 coding sequence (NCBI Reference Sequence NM_001136) was synthesized (Genscript) and subcloned into pCI-neo (Promega) to generate the vector pCIneo-AGER. This plasmid was subsequently used to generate full-length and truncated AGER clones with or without point mutations R366A and Q367A, S391A, S391Q, S391P, S399A, S400A, T401A relative to the reference protein sequence NP_001127. The no-STED mutation was generated using a specific oligonucleotide encoding the residues (QPKGPQAGQGGAGGP) relative to residues 390-404 of Homo sapiens AGER. The human/mouse chimera was generated using a specific oligonucleotide encoding the Mus musculus residues (QSEEAEMPENGAGGP) relative to residues 390-404 of Homo sapiens AGER. Truncations were generated using specific oligonucleotides to encode a STOP codon and delete the residues following the mutation. For example, S391X was truncated after the codon encoding Q390, in which 391 S and residues 392-404 were not present in the resultant plasmid, rather than generating a STOP codon by point mutation of the full-length $A G E R$ sequence. The coding sequence of mCherry was amplified from the vector pmCherry-C1 (Clontech). The resultant PCR product was then used as a template for a second PCR using primers that added the RAGE signal peptide (and 3 residues after the 
predicted cleavage site [residue 22] 1-25). The PCR product generated was cloned into pCIneo to generate pCIneo-SP-mCherry. For transmembrane RAGE constructs, portions of the AGER gene encoding residues 343-404 or 338-361 were amplified from pCIneo-AGER WT or the S391A mutant template and subcloned into the $3^{\prime}$ end of pCI-neo-SP-mCherry. For cytoplasmic construct expression, the coding sequence of mCherry was amplified from the vector pmCherry$\mathrm{C} 1$ and subcloned into pCI-neo to generate pCI-neo-mCherry. Portions of the WT or S391A AGER gene encoding residues 362-404 and 370-404 were amplified from the vector pCIneo-AGER and subcloned into pCI-neo-mCherry to generate cytoplasmic RAGE constructs. In each case, all sequences of the inserts were confirmed by DNA sequencing (Micromon, Monash University).

$B R E T$. To explore the potential for a heteromeric $\mathrm{AT}_{1}$ receptorRAGE complex, we used BRET, an established technology for studying GPCR-protein proximity in live cells $(48,49)$. The $\mathrm{AT}_{1}$ receptor, RAGE, and interacting proteins were linked to a bioluminescent donor enzyme, Rluc8 or Nluc, or an acceptor fluorophore, Venus or mCherry. Whether the donor or acceptor is fused to the $\mathrm{N}$ - or C-terminus is denoted by it being written before or after the protein of interest, respectively (e.g., Nluc-AT $\mathrm{V}_{1}$ s. $\mathrm{AT}_{1}$-Nluc). If in close proximity $(<10$ $\mathrm{nm}$ ), energy resulting from the rapid oxidation of a cell-permeable coelenterazine or furimazine substrate by the donor can transfer to the acceptor, which in turn fluoresces at a longer characteristic wavelength. $\mathrm{AT}_{1}$ and $\mathrm{AT}_{1}$-Rluc cDNA was provided by Walter Thomas (University of Queensland, St. Lucia, Brisbane, Australia). The Rluc coding region was replaced with Rluc8 cDNA from pcDNA3.1-Rluc8 provided by Andreas Loening and Sanjiv Gambhir (Stanford University, Stanford, California, USA) (50). Nluc-AT ${ }_{1}$ was provided by Promega, and $\mathrm{AT}_{1}$-Nluc was generated from $\mathrm{AT}_{1}-\mathrm{Rluc} 8$ by replacing Rluc8 with Nluc that was synthesized by GeneArt (Thermo Fisher Scientific).

For receptor-HIT (receptor-heteromer investigation technology) assays (Receptor-HIT, Dimerix Ltd.), RAGE-Rluc8 and $\beta$-arrestin2Venus were expressed with untagged $\mathrm{AT}_{1}$. In this system, ligandinduced BRET is indicative of recruitment of $\beta$-arrestin2-Venus proximal to RAGE-Rluc8, thereby indicating close proximity between RAGE and activated $\mathrm{AT}_{1}$. The $\beta$-arrestin2-Venus cDNA construct was prepared previously from pCS2-Venus, provided by Atsushi Miyawaki (RIKEN Brain Science Institute, Wako City, Japan) (50). RAGE cDNA was amplified by PCR from pCI-neo-Ager to remove the stop codon and ligated into pcDNA3-Venus and pcDNA3-Rluc8. As a control for $\beta$-arrestin recruitment, the cDNA clone for CCR4 was obtained from the cDNA Resource Center (Bloomsburg, Pennsylvania, USA). CCR4 cDNA was amplified by PCR to remove the stop codon and ligated into pcDNA3-Rluc8. G $\alpha_{i}$-Nluc cDNA was generated by GeneArt (Thermo Fisher Scientific, Germany). $\mathrm{G} \gamma_{2}$-Venus was provided by Martina Kocan (The Florey Institute of Neuroscience and Mental Health, Parkville, Melbourne, Australia).

To explore changes in the subcellular localization of RAGE induced following ligand binding, BRET between RAGE and subcellular marker proteins was also explored. The plasma membrane marker Venus-Kras, as well as the subcellular compartment marker RabGTPases (Rabs) Venus-Rab5a (early endosomes), Venus-Rab7a (late endosomes/ lysosomes), and Venus-Rab11a (recycling endosomes), were provided by Nevin Lambert (Georgia Regents University/Augusta University, Augusta, Georgia, USA). Rab1 (ER trafficking to the cis-Golgi), Rab4 (early endosome recycling), Rab6 (cis-Golgi), Rab8 (trans-Golgi net- work to the plasma membrane), and Rab9 (late endosome trafficking to the trans-Golgi network) were synthesized and subcloned by GeneArt into pcDNA3-Venus, as described previously (26).

Plasmids were transiently coexpressed in human embryonic kidney (HEK) 293FT cells, and BRET measurements were taken at $37^{\circ} \mathrm{C}$ using either a POLARstar Omega or LUMIstar Omega plate reader (BMG LABTECH) with 460-490 nm (“donor emission”) and 520-550 $\mathrm{nm}$ ("acceptor emission") filters; a CLARIOstar plate reader (BMG LABTECH) with 420-480 nm (donor emission) and 520-620 nm (acceptor emission) filters; or a VICTOR Light plate reader (PerkinElmer) with 400-475 nm (donor emission) and 520- to 540-nm (acceptor emission) filters. For assays using the mCherry constructs, the CLARIOstar plate reader was used with 420- to 480-nm donor emission and 580- to 680-nm acceptor emission. Note that BRET ratios differ when measured by different instruments because of the differences in sensitivity, filter configuration, and gain settings. Consequently, the same instrument setup was used to generate multiple repeats for a given data set. For the saturation assay, the BRET ratio was calculated by subtracting the ratio of the acceptor emission over the donor emission for a cell sample expressing Rluc8-tagged protein alone from the same ratio for a cell sample expressing both Rluc8 and Venus-tagged proteins. For all other BRET assays, the ligand-induced BRET signal was calculated by subtracting the ratio of the acceptor emission over the donor emission for a vehicle-treated cell sample from the same ratio for a second aliquot of the same cells treated with an agonist. For BRET kinetic assays, the final pretreatment reading is presented at the zero time point (time of ligand/vehicle addition). For the saturation assay, fluorescence after light excitation was measured on an EnVision 2102 multilabel plate reader (PerkinElmer) using a 485/14 excitation filter, a 535/25 emission filter, and a D505 mirror. The fluorescence/luminescence ratio was generated by dividing the fluorescence values in AU (obtained with the EnVision) by the luminescence values, also in AU (obtained as part of the BRET assay), as described previously (51). The ligands used were Ang II (MilliporeSigma), CCL22 (Peprotech), soluble RAGE, TAT-mCherry, and WT or S391A TAT-mCherryRAGE $_{362-404}$, as described below. BRET substrates used were EnduRen for Rluc8 assays and caged furimazine for Nluc assays (Promega).

Studies with cell-penetrating oligopeptides. Oligonucleotide primers encoding a deca-histidine sequence followed by residues 47-57 of the HIV-1 TAT protein were fused to the N-terminus of mCherry, mCherry-RAGE ${ }_{362-404}$, and mCherry-S391A-RAGE ${ }_{362-404}$ to generate TAT-mCherry, TAT-mCherry-RAGE ${ }_{362-404}$, and TAT-mCherry-S391ARAGE $_{362-404}$. These constructs were subcloned into the pCOLD IV vector (Takara Bio). All constructs were confirmed by DNA sequencing (Micromon, Monash University).

Cell-penetrating oligopeptides (TAT-mCherry, TAT-mCherryRAGE $_{362-404}$, and TAT-mCherry-S391A-RAGE ${ }_{362-404}$ ) were generated by transformation of TAT-encoding plasmids into the Escherichia coli strain ClearColi BL21 (DE3) (Lucigen). A single transformed colony was inoculated into $50 \mathrm{ml} 2 \mathrm{YT}$ medium containing $100 \mu \mathrm{g} / \mathrm{ml}$ ampicillin and the culture grown overnight at $37^{\circ} \mathrm{C}$ with shaking at 250 $\mathrm{rpm}$. Overnight culture $(10 \mathrm{ml})$ was used to inoculate 1 liter of $2 \mathrm{YT}$ medium containing $100 \mu \mathrm{g} / \mathrm{ml}$ ampicillin and the culture grown at $37^{\circ} \mathrm{C}$ with shaking at $250 \mathrm{rpm}$. When the cultures reached an $\mathrm{OD}_{600}$ of 0.8 , the temperature was shifted to $15^{\circ} \mathrm{C}$ for 30 minutes, after which protein expression was induced by the addition of $0.5 \mathrm{mM}$ isopropyl $\beta$-D-1-thiogalactopyranoside (IPTG). Following the addition of IPTG, the cultures were grown for approximately 16 hours at $15^{\circ} \mathrm{C}$ with shak- 
ing at $250 \mathrm{rpm}$. Cells were harvested by centrifugation $\left(3500 \mathrm{~g}, 4^{\circ} \mathrm{C}\right.$, $20 \mathrm{~min}$ ) and resuspended in ice-cold lysis buffer (50 mM Tris, $\mathrm{pH}$ 7.4, $300 \mathrm{mM} \mathrm{NaCl}, 10 \mathrm{mM}$ imidazole, $5 \mathrm{mM} \beta$-mercaptoethanol). The resuspended cells were sonicated, and insoluble material was pelleted by centrifugation $\left(13,000 \mathrm{~g}, 4^{\circ} \mathrm{C}, 30 \mathrm{~min}\right)$. The supernatant was filtered $(0.2 \mu \mathrm{m})$ and applied onto a gravity flow column with a 1-ml Ni-NTA agarose resin bed volume (QIAGEN) preequilibrated with lysis buffer. After the supernatant was passed over the resin, the column was washed with $100 \mathrm{ml}$ wash buffer $(20 \mathrm{mM}$ imidazole, $50 \mathrm{mM}$ Tris, pH 7.4, $150 \mathrm{mM} \mathrm{NaCl}, 5 \mathrm{mM} \beta$-mercaptoethanol). Histagged TAT-mCherry and TAT-RAGE peptides were eluted from the resin using elution buffer ( $250 \mathrm{mM}$ imidazole, $50 \mathrm{mM}$ Tris, $\mathrm{pH}$ 7.4, 150 $\mathrm{mM} \mathrm{NaCl}, 5 \mathrm{mM} \beta$-mercaptoethanol). The eluted fractions containing protein were pooled and quantified by NanoDrop spectrophotometry (Thermo Fisher Scientific) and BCA Protein Assay (Pierce, Thermo Fisher Scientific). For all in vivo applications, the peptides were buffer exchanged into sterile saline solution and the protein concentration recalculated. Cultured cells were treated with oligopeptides at a dose of $0.4 \mathrm{ng} / \mathrm{ml}$, unless otherwise stated. Mice received respective peptides at a dose of $10 \mu \mathrm{g} / \mathrm{kg}$ i.p. every other day for 10 weeks.

Statistics. Continuous data are expressed as the mean \pm SD. Differences in the mean among groups were compared using 2-way ANOVA. Pairwise multiple comparisons were made with Student-NewmanKeuls post hoc analysis to detect significant differences between groups, using Bonferroni's correction for multiple comparisons. A $P$ value of less than 0.05 was considered statistically significant.
Study approval. All experiments were approved by the animal ethics committee of the Alfred Medical Research Precinct and conducted in accordance with the Guide for the Care and Use of Laboratory Animals (NIH publication 85-23; National Academies Press, 1996).

\section{Author contributions}

RJP, CT, CJR, DT, AD, MS, and MCT performed the cell culture and in vivo studies and analyzed the data. OH performed the dynamic flow assays. KAJD provided the Ager-KO and Ager/ApoeDKO mice. YW and JG performed the angiotensin infusion studies. RMS, RA, EKMJ, and KDGP performed the BRET research and analyzed the data. MCT, MEC, and KDGP wrote the manuscript, and all authors contributed to its final version.

\section{Acknowledgments}

This work was supported by Australian National Health and Medical Research Council (NHMRC) project grant 1081013. MCT, KJD, and MEC received Senior Research Fellowships from the Australian NHMRC (grants 1107555, 1059124, and 1078808, respectively), and KDGP is an NHMRC RD Wright Biomedical Research Fellow (grant 1085842).

Address correspondence to: Merlin C. Thomas; Department of Diabetes, Central Clinical School, Monash University, Melbourne, VIC 3004, Australia. Phone: 61.3.9903.0099; Email: Merlin. Thomas@monash.edu.
1. Ruiz-Ortega M, Lorenzo O, Rupérez M, König S, Wittig B, Egido J. Angiotensin II activates nuclear transcription factor kappaB through AT(1) and AT(2) in vascular smooth muscle cells: molecular mechanisms. Circ Res. 2000;86(12):1266-1272.

2. Li XC, Zhuo JL. Nuclear factor-kappaB as a hormonal intracellular signaling molecule: focus on angiotensin II-induced cardiovascular and renal injury. Curr Opin Nephrol Hypertens. 2008;17(1):37-43.

3. Douillette A, et al. The proinflammatory actions of angiotensin II are dependent on $\mathrm{p} 65$ phosphorylation by the IkappaB kinase complex. J Biol Chem. 2006;281(19):13275-13284.

4. Candido R, et al. Prevention of accelerated atherosclerosis by angiotensin-converting enzyme inhibition in diabetic apolipoprotein E-deficient mice. Circulation. 2002;106(2):246-253.

5. Knowles JW, Reddick RL, Jennette JC, Shesely EG, Smithies O, Maeda N. Enhanced atherosclerosis and kidney dysfunction in $\mathrm{eNOS}^{-/-} \mathrm{Apoe}^{-/-}$ mice are ameliorated by enalapril treatment. JClin Invest. 2000;105(4):451-458.

6. Benigni A, et al. Disruption of the Ang II type 1 receptor promotes longevity in mice. JClin Invest. 2009;119(3):524-530.

7. Candido R, et al. Irbesartan but not amlodipine suppresses diabetes-associated atherosclerosis. Circulation. 2004;109(12):1536-1542.

8. Seta K, Nanamori M, Modrall JG, Neubig RR, Sadoshima J. AT1 receptor mutant lacking heterotrimeric $G$ protein coupling activates the SrcRas-ERK pathway without nuclear translocation of ERKs. J Biol Chem. 2002;277(11):9268-9277.

9. Domazet I, et al. Characterization of Angioten- sin II Molecular Determinants Involved in AT1 Receptor Functional Selectivity. Mol Pharmacol. 2015;87(6):982-995.

10. Ballinger ML, Thomas MC, Nigro J, Ivey ME, Dilley RJ, Little PJ. Glycated and carboxymethylated proteins do not directly activate human vascular smooth muscle cells. Kidney Int. 2005;68(6):2756-2765.

11. Deane R, et al. RAGE mediates amyloid-beta peptide transport across the blood-brain barrier and accumulation in brain. Nat Med. 2003;9(7):907-913

12. Taguchi A, et al. Blockade of RAGE-amphoterin signalling suppresses tumour growth and metastases. Nature. 2000;405(6784):354-360.

13. Goldin A, Beckman JA, Schmidt AM, Creager MA. Advanced glycation end products: sparking the development of diabetic vascular injury. Circulation. 2006;114(6):597-605.

14. Soro-Paavonen A, et al. Receptor for advanced glycation end products (RAGE) deficiency attenuates the development of atherosclerosis in diabetes. Diabetes. 2008;57(9):2461-2469.

15. Schmidt AM, Yan SD, Wautier JL, Stern D. Activation of receptor for advanced glycation end products: a mechanism for chronic vascular dysfunction in diabetic vasculopathy and atherosclerosis. Circ Res. 1999;84(5):489-497.

16. Park L, et al. Suppression of accelerated diabetic atherosclerosis by the soluble receptor for advanced glycation endproducts. Nat Med. 1998;4(9):1025-1031.

17. Zhou Z, et al. Receptor for AGE (RAGE) mediates neointimal formation in response to arterial injury. Circulation. 2003;107(17):2238-2243.
18. Bierhaus A, et al. Diabetes-associated sustained activation of the transcription factor nuclear factor-kappaB. Diabetes. 2001;50(12):2792-2808.

19. Thomas MC, et al. Genetic Ace 2 deficiency accentuates vascular inflammation and atherosclerosis in the ApoE knockout mouse. Circ Res. 2010;107(7):888-897.

20. Paigen B, Morrow A, Brandon C, Mitchell D, Holmes P. Variation in susceptibility to atherosclerosis among inbred strains of mice. Atherosclerosis. 1985;57(1):65-73.

21. Overland AC, Insel PA. Heterotrimeric $G$ proteins directly regulate MMP14/membrane type-1 matrix metalloprotease: a novel mechanism for GPCR-EGFR transactivation. J Biol Chem. 2015;290(16):9941-9947.

22. Hudson BI, et al. Interaction of the RAGE cytoplasmic domain with diaphanous-1 is required for ligand-stimulated cellular migration through activation of Rac1 and Cdc42. J Biol Chem. 2008;283(49):34457-34468.

23. Porrello ER, et al. Heteromerization of angiotensin receptors changes trafficking and arrestin recruitment profiles. Cell Signal. 2011;23(11):1767-1776.

24. Johnstone EK, Pfleger KD. Receptor-Heteromer Investigation Technology and its application using BRET. Front Endocrinol (Lausanne). 2012;3:101.

25. Jaeger WC, Armstrong SP, Hill SJ, Pfleger KD. Biophysical detection of diversity and bias in GPCR function. Front Endocrinol (Lausanne). 2014;5:26.

26. Tiulpakov A, et al. Mutations of vasopressin receptor 2 including novel L312S have differential effects on trafficking. Mol Endocrinol. 
2016;30(8):889-904.

27. Shaw SS, Schmidt AM, Banes AK, Wang X, Stern DM, Marrero MB. S100B-RAGE-mediated augmentation of angiotensin II-induced activation of JAK2 in vascular smooth muscle cells is dependent on PLD2. Diabetes. 2003;52(9):2381-2388.

28. Doughan AK, Harrison DG, Dikalov SI. Molecular mechanisms of angiotensin II-mediated mitochondrial dysfunction: linking mitochondrial oxidative damage and vascular endothelial dysfunction. Circ Res. 2008;102(4):488-496.

29. Wautier MP, Chappey O, Corda S, Stern DM, Schmidt AM, Wautier JL. Activation of NADPH oxidase by AGE links oxidant stress to altered gene expression via RAGE. Am J Physiol Endocrinol Metab. 2001;280(5):E685-E694.

30. Kamioka M, et al. Blockade of renin-angiotensin system attenuates advanced glycation end products-mediated signaling pathways. J Atheroscler Thromb. 2010;17(6):590-600.

31. Fukami K, et al. AGEs activate mesangial TGF-beta-Smad signaling via an angiotensin II type I receptor interaction. Kidney Int. 2004;66(6):2137-2147.

32. Huang JS, Guh JY, Chen HC, Hung WC, Lai YH, Chuang LY. Role of receptor for advanced glycation end-product (RAGE) and the JAK/ STAT-signaling pathway in AGE-induced collagen production in NRK-49F cells. J Cell Biochem. 2001;81(1):102-113.

33. Fujita M, et al. Blockade of angiotensin II receptors reduces the expression of receptors for advanced glycation end products in human endothelial cells. Arterioscler Thromb Vasc Biol. 2006;26(10):e138-e142.

34. Thomas MC, et al. Interactions between renin angiotensin system and advanced glycation in the kidney. J Am Soc Nephrol.
2005;16(10):2976-2984.

35. Sakaguchi M, et al. TIRAP, an adaptor protein for TLR2/4, transduces a signal from RAGE phosphorylated upon ligand binding. PLOS ONE. 2011;6(8):e23132.

36. Touré $\mathrm{F}$, et al. Formin mDia1 mediates vascular remodeling via integration of oxidative and signal transduction pathways. Circ Res. 2012;110(10):1279-1293.

37. Eng CH, Huckaba TM, Gundersen GG. The formin mDia regulates GSK3beta through novel PKCs to promote microtubule stabilization but not MTOC reorientation in migrating fibroblasts. Mol Biol Cell. 2006;17(12):5004-5016.

38. García-Hoz C, et al. Protein kinase C (PKC) $\zeta$ mediated Gaq stimulation of ERK5 protein pathway in cardiomyocytes and cardiac fibroblasts. J Biol Chem. 2012;287(10):7792-7802.

39. Rai V, et al. Signal transduction in receptor for advanced glycation end products (RAGE): solution structure of C-terminal rage (ctRAGE) and its binding to mDia1. J Biol Chem. 2012;287(7):5133-5144.

40. Tanji M, et al. mDia1 targets v-Src to the cell periphery and facilitates cell transformation, tumorigenesis, and invasion. Mol Cell Biol. 2010;30(19):4604-4615.

41. Choudhary S, Lu M, Cui R, Brasier AR. Involvement of a novel Rac/RhoA guanosine triphosphatase-nuclear factor-kappaB inducing kinase signaling pathway mediating angiotensin II-induced RelA transactivation. Mol Endocrinol. 2007;21(9):2203-2217.

42. Thomas MC, Tikellis C. Losing control: positive and negative feedback in the renin angiotensin system. Curr Hypertens Rev. 2009;5(3):222-226.

43. Constien R, et al. Characterization of a novel EGFP reporter mouse to monitor Cre recombina- tion as demonstrated by a Tie 2 Cre mouse line. Genesis. 2001;30(1):36-44.

44. Ray R, Juranek JK, Rai V. RAGE axis in neuroinflammation, neurodegeneration and its emerging role in the pathogenesis of amyotrophic lateral sclerosis. Neurosci Biobehav Rev. 2016;62:48-55.

45. Watson AM, et al. Alagebrium reduces glomerular fibrogenesis and inflammation beyond preventing RAGE activation in diabetic apolipoprotein E knockout mice. Diabetes. 2012;61(8):2105-2113.

46. Tikellis C, et al. Activation of the renin-angiotensin system mediates the effects of dietary salt intake on atherogenesis in the apolipoprotein $\mathrm{E}$ knockout mouse. Hypertension. 2012;60(1):98-105.

47. Koulis $\mathrm{C}$, et al. Role of bone-marrow- and nonbone-marrow-derived receptor for advanced glycation end-products (RAGE) in a mouse model of diabetes-associated atherosclerosis. Clin Sci. 2014;127(7):485-497.

48. Pfleger KD, Eidne KA. Illuminating insights into protein-protein interactions using bioluminescence resonance energy transfer (BRET). Nat Methods. 2006;3(3):165-174.

49. Pfleger KD, Seeber RM, Eidne KA. Bioluminescence resonance energy transfer (BRET) for the real-time detection of protein-protein interactions. Nat Protoc. 2006;1(1):337-345.

50. Kocan M, See HB, Seeber RM, Eidne KA, Pfleger KD. Demonstration of improvements to the bioluminescence resonance energy transfer (BRET) technology for the monitoring of $G$ protein-coupled receptors in live cells. J Biomol Screen. 2008;13(9):888-898.

51. Mustafa S, et al. Identification and profiling of novel $\alpha 1 \mathrm{~A}$-adrenoceptor-CXC chemokine receptor 2 heteromer. J Biol Chem. 2012;287(16):12952-12965. 